\title{
Lebesgue orbit equivalence of multidimensional Borel flows: A picturebook of tilings
}

\author{
KONSTANTIN SLUTSKY \\ Department of Mathematics, Statistics and Computer Science, \\ University of Illinois at Chicago, 322 Science and Engineering Offices (M/C 249), \\ 851 S. Morgan Street, Chicago, IL 60607-7045, USA \\ (e-mail: kslutsky@gmail.com)
}

(Received 7 July 2015 and accepted in revised form 8 October 2015)

\begin{abstract}
The main result of the paper is classification of free multidimensional Borel flows up to Lebesgue orbit equivalence, by which we mean an orbit equivalence that preserves the Lebesgue measure on each orbit. Two non-smooth $\mathbb{R}^{d}$-flows are shown to be Lebesgue orbit equivalent if and only if they admit the same number of invariant ergodic probability measures.
\end{abstract}

\section{Introduction}

1.1. Orbit equivalences. The core concept for this paper is orbit equivalence of actions. Since we work in the framework of descriptive set theory, we consider Borel actions of Polish groups $\dagger$ on standard $\ddagger$ Borel spaces. Two group actions $G \curvearrowright X$ and $H \curvearrowright Y$ are orbit equivalent $(\mathrm{OE})$ if there exists a Borel bijection $\phi: X \rightarrow Y$ which sends orbits onto orbits: that is, if

$$
\phi\left(\operatorname{Orb}_{G}(x)\right)=\operatorname{Orb}_{H}(\phi(x)) \text { for all } x \in X .
$$

This notion is, perhaps, better known in the framework of ergodic theory, where phase spaces $X$ and $Y$ are endowed with invariant probability measures which the orbit equivalence is assumed to preserve. Our set up is different in two aspects. We do not fix any measures on the phase spaces, therefore potentially increasing the choice of orbit equivalence maps. On the other hand, contrary to the ergodic theoretical case where functions need to be defined almost everywhere, we require our maps $\phi: X \rightarrow Y$ to be defined on each and every point. In this sense, the descriptive theoretical set up is more restrictive.

\footnotetext{
$\dagger$ A topological group is Polish if its topology is Polish, i.e., it is separable and metrizable by a complete metric. $\$$ A standard Borel space is a set $X$ together with a distinguished $\sigma$-algebra $\Sigma$ such that for some Polish topology on $X$ the $\sigma$-algebra $\Sigma$ coincides with the family of Borel sets.
} 
The notion of $\mathrm{OE}$ in the above form is arguably better suited for actions of discrete groups. One of the high points in that area is the classification of hyperfinite equivalence relations up to orbit equivalence by Dougherty et al [DJK94, Theorem 9.1] based on an earlier work of Nadkarni [Nad90]. First, let us recall that with any action $G \curvearrowright X$ we may associate the orbit equivalence relation on $X$ (denoted by $E_{X}^{G}$, or just by $E_{X}$, when the group is understood) by declaring two points to be equivalent whenever they are in the same orbit of the action. We say that an equivalence relation is countable if each equivalence class is countable (but note that there will typically be an uncountable number of classes), and an equivalence relation is finite if every equivalence class is finite. A countable Borel equivalence relation is hyperfinite if it is an increasing union of finite Borel equivalence relations. Given a countable Borel equivalence relation $E \subseteq X \times X$, a measure $\mu$ on $X$ is said to be $E$-invariant if every Borel automorphism $\theta: X \rightarrow X$ of $E$ preserves $\mu$. A measure is ergodic with respect to $E$ if every $E$-invariant subset of $X$ is either null or conull. For the sake of brevity, a probability invariant ergodic measure is called a pie measure. A countable equivalence relation is aperiodic if all of its classes are infinite. Finally, recall that a countable equivalence relation is smooth if it admits a Borel transversal-a Borel set that intersects every equivalence class in exactly one point. Having introduced all the necessary terminology, the Dougherty-Jackson-Kechris classification (we later refer to it as DJK classification for short) of hyperfinite Borel equivalence relations can be stated by the following theorem.

THEOREM. (Dougherty-Jackson-Kechris) Two non-smooth aperiodic hyperfinite Borel equivalence relations are isomorphic if and only if cardinalities of the sets of pie measures are the same.

We recall that hyperfinite equivalence relations are exactly the orbit equivalence relations arising from Borel actions of the group of integers $\mathbb{Z}$ (see, for instance, [DJK94, Theorem 5.1]). The situation changes drastically when one considers locally compact nondiscrete groups. All free non-smooth Borel $\mathbb{R}$-flows are orbit equivalent. In fact, a much stronger result is true. An orbit equivalence $\phi: X \rightarrow Y$ between two free actions $\mathbb{R} \curvearrowright X$ and $\mathbb{R} \curvearrowright Y$ gives rise to a cocycle $\dagger$

$$
f: \mathbb{R} \times X \rightarrow \mathbb{R} \quad f(r, x) \text { is such that } \phi(x)+f(r, x)=\phi(x+r) .
$$

A time-change equivalence between free actions $\mathbb{R} \curvearrowright X$ and $\mathbb{R} \curvearrowright Y$ is an $\mathrm{OE} \phi: X \rightarrow Y$ such that for each $x \in X$ the function $f(\cdot, x): \mathbb{R} \rightarrow \mathbb{R}$ is a homeomorphism $\ddagger$. This is a substantial strengthening of the notion of orbit equivalence. Nevertheless, as proved by Miller and Rosendal [MR10], in the descriptive set theoretic set-up the world of free $\mathbb{R}$-flows collapses with respect to time-change equivalence.

Theorem. (Miller-Rosendal) Any two non-smooth free $\mathbb{R}$-flows are time-change equivalent.

$\dagger$ For abelian groups the action is denoted additively. For instance, $x+r$ below means the action of $r \in \mathbb{R}$ upon the element $x \in X$.

¥ One may even require a stronger condition of $f(\cdot, x)$ being a $C^{\infty}$-diffeomorphism. The theorem of Miller and Rosendal remains valid in this case. 
The difference between continuous and discrete worlds lies in the fact that continuous groups have a lot more non-trivial structures on them. The obvious one is topology. Whenever we have a free action $G \curvearrowright X$ and an orbit $\mathcal{O} \subseteq X$, we may transfer the topology from $G$ onto $\mathcal{O}$ using the correspondence $G \ni g \mapsto g x \in \mathcal{O}$ for any chosen $x \in \mathcal{O}$. If groups $G$ and $H$ are discrete, any $\mathrm{OE}$ between their free actions respects the topology: restricted onto any orbit $\mathcal{O} \subseteq X, \phi: \mathcal{O} \rightarrow \phi(\mathcal{O})$ is a homeomorphism. When the topology on $\mathcal{O}$ is not discrete, the map $\phi: \mathcal{O} \rightarrow \phi(\mathcal{O})$ has no reason to preserve the topology and, when this is imposed as an additional assumption on $\phi$, one recovers the concept of timechange equivalence.

Another structure possessed by all locally compact groups is Haar measure. Being invariant, it can also be transferred $\dagger$ onto any orbit of a free action $G \curvearrowright X$. Again, if $G$ and $H$ are discrete (and if one takes counting Haar measures), any OE map $\phi: X \rightarrow Y$ restricts to a measure preserving isomorphism between orbits. When $G$ and $H$ are continuous, this may no longer be the case. This turns out to be an obstacle for cardinality of the set of pie measures to be an invariant of the $\mathrm{OE}$ between non-discrete locally compact group actions and is responsible for the failure of the analog of DJK classification.

1.2. Lebesgue orbit equivalence. The paper is concerned mainly with free actions of Euclidean spaces $\mathbb{R}^{d} \curvearrowright X$ on standard Borel spaces. Two such actions $\mathbb{R}^{d} \curvearrowright X$ and $\mathbb{R}^{d} \curvearrowright Y$ are Lebesgue orbit equivalent (LOE) if there exists an OE $\phi: X \rightarrow Y$ which preserves the Lebesgue measure on each orbit $\ddagger$. In an ergodic theoretical set-up, i.e., when $X$ and $Y$ are endowed with probability invariant measures and the map $\phi$ needs to be defined almost everywhere, this notion seems to have appeared for the first time in the work of Krengel [Kre76], in which the following theorem was proved.

THEOREM. (Krengel) Free ergodic flows $\mathbb{R} \curvearrowright X$ and $\mathbb{R} \curvearrowright Y$ are always LOE.

Still within the framework of ergodic theory, this has later been generalized by Rudolph [Rud79] to free actions of $\mathbb{R}^{d}$. In fact, Rudolph proved a much stronger result. Namely, for $d \geq 2$ an OE map $\phi: X \rightarrow Y$ may be assumed to be both Lebesgue measure preserving and a homeomorphism when restricted to any orbit.

1.3. Main results. In the present paper we prove an analog of DJK classification for free $\mathbb{R}^{d}$-flows.

THEOREM 9.1. Free non-smooth Borel $\mathbb{R}^{d}$-flows are Lebesgue orbit equivalent if and only if cardinalities of the sets of pie measures are the same.

The structure of the paper is as follows. Section 2 provides a different proof of a theorem due to Conley which guarantees existence of cocompact cross sections. Section 3 introduces various notions of orbit equivalences which preserve measures between orbits and $\S 4$ shows a correspondence between invariant measures on a phase space and on a cocompact cross section.

$\dagger$ Since we consider left actions one needs to take a right Haar measure. More on this in $\$ 3$.

$\$$ A rigorous definition can be found in $\S 3$. 
Remaining sections deal with $\mathbb{R}^{d}$-flows and prove various parts of the main result. In $\S 5$ we introduce rectangular tilings, §6 proves a version of Rokhlin's lemma that works for all invariant measures at the same time, and $\$ 7$ employs this result together with a tiling method due to Rudolph [Rud88] to construct a LOE between invariant subsets of uniformly full measures. Section 8 then deals with the complementary case of flows with no invariant measures, and in $\S 9$ we finally prove the main theorem.

\section{Cocompact cross sections}

Let $G$ be a locally compact Polish group acting in a Borel way on a standard Borel space $X$. In this section the action is not assumed to be free. A cross section for $G \curvearrowright X$ is a Borel set $\mathcal{C} \subseteq X$ that intersects every orbit of the action, i.e., $G \cdot \mathcal{C}=X$, and is such that, for some neighborhood of the identity $U \subseteq G$,

$$
U \cdot x \cap U \cdot y=\varnothing \quad \text { whenever } x, y \in \mathcal{C} \text { are distinct. }
$$

We shall typically assume $U$ to be symmetric and compact. When for a set $U$ the cross section $\mathcal{C}$ satisfies (1), we say that the cross section is $U$-lacunary. Frequently, the definition of a cross section is relaxed by omitting the lacunarity condition and requiring the countability of intersections with orbits instead but, since all the cross sections in our work will be lacunary, we choose to adopt the stronger definition.

Lacunarity says that distinct points within an orbit are never 'too close' to each other. The complementary notion of not having 'large gaps' within orbits is called cocompactness. A cross section $\mathcal{C}$ is cocompact if there exists a compact neighborhood of the identity $V \subseteq G$ such that $V \cdot \mathcal{C}=X$, and $\mathcal{C}$ is said to be $V$-cocompact in that case. Finally, we say that a cross section $\mathcal{C}$ is a maximal $U$-lacunary cross section if it is $U$-lacunary and, for any $z \in X \backslash \mathcal{C}$, the set $\mathcal{C} \cup\{z\}$ is no longer a $U$-lacunary cross section. When $U$ is a symmetric neighborhood of the identity, a $U$-lacunary cross section is maximal if and only if it is $U^{2}$-cocompact.

To illustrate this concept consider, for instance, the case $G=\mathbb{R}^{2}$ and suppose, additionally, that the action $\mathbb{R}^{2} \curvearrowright X$ is free. We may therefore identify each orbit with an affine $\dagger$ copy of the plane. Take $U$ to be a ball of radius $r$ around the origin in $\mathbb{R}^{2}$. A cross section $\mathcal{C} \subset X$ is $U$-lacunary if and only if balls of radius $r$ around distinct points in $\mathcal{C}$ do not intersect, see Figure 1 . It is maximal $U$-lacunary if, moreover, any point in the orbit is at most $2 r$ from a point in $\mathcal{C}$.

A theorem of Kechris [Kec92, Corollary 1.2] establishes existence of $U$-lacunary cross sections for any action $G \curvearrowright X$ of a locally compact group and any given compact neighborhood of the identity $U \subseteq G$. Conley proved that one can always find a cocompact cross section.

THEOREM. (Conley) Any Borel action of a locally compact group admits a cocompact cross section.

$\dagger$ By affine we mean a plane with no distinguished origin. It nevertheless carries all the structures from $\mathbb{R}^{2}$ which are translation invariant: Euclidean distance, Lebesgue measure, etc. 


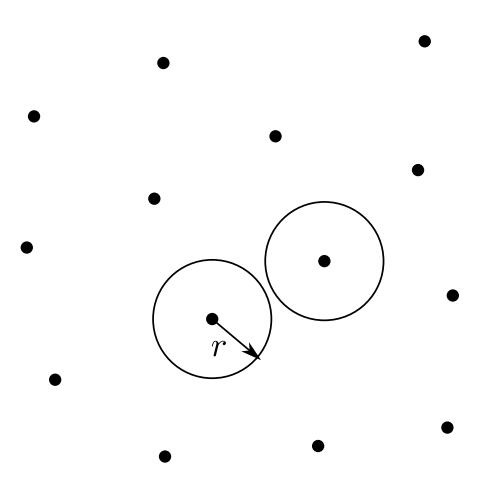

FIGURE 1. Cocompact cross section.

Conley's argument uses $G_{0}$-dichotomy. For the purpose of completeness, we present, in this section, an elementary construction of enlarging any cross section into a maximal one.

Let $\mathcal{C} \subseteq X$ be a cross section, and let $W \subseteq G$ be a compact symmetric neighborhood of the identity. We turn $\mathcal{C}$ into a Borel graph with the set of edges $F_{W}$ by putting an edge between $x$ and $y$ whenever there exists $g \in W$ such that $g x=y$ : that is,

$$
F_{W}=\{(x, y) \in \mathcal{C} \times \mathcal{C}: \exists g \in W g x=y\} .
$$

Lacunarity of $\mathcal{C}$ ensures that the degree of any vertex in $F_{W}$ is finite (in fact, the degree is uniformly bounded). A subset $\mathcal{A} \subseteq \mathcal{C}$ is said to be $F_{W}$-independent if there are no edges between its points: that is,

$$
x \neq y \Longrightarrow(x, y) \notin F_{W} \quad \text { for all } x, y \in \mathcal{A} .
$$

The proof of [JKL02, Lemma 1.17] shows that $\mathcal{C}$ can be written as a disjoint union of a countable number of Borel $F_{W}$-independent subsets. We reproduce the argument for the reader's convenience and refer to [KST99] for the background on Borel combinatorics.

LEMMA 2.1. Let $\mathcal{C} \subseteq X$ be a cross section and let $W \subseteq G$ be a compact symmetric neighborhood of the identity. There exist Borel $F_{W}$-independent subsets $\mathcal{A}_{n} \subseteq \mathcal{C}$ such that $\mathcal{C}=\bigsqcup_{n \in \mathbb{N}} \mathcal{A}_{n}$.

Proof. Let $\left(Z_{n}\right)_{n=1}^{\infty}$ be a countable family of Borel subsets of $\mathcal{C}$ which separates points and which is closed under finite intersections. Since $F_{W}$ is locally finite, for any $x \in \mathcal{C}$ there exist $n$ such that $F_{W}[x] \cap Z_{n}=\{x\}$, where

$$
F_{W}[x]=\left\{y \in \mathcal{C}:(x, y) \in F_{W}\right\} .
$$

Let $\phi: \mathcal{C} \rightarrow \mathbb{N}$ be given by

$$
\phi(x)=\min \left\{n \in \mathbb{N}: F_{W}[x] \cap Z_{n}=\{x\}\right\} .
$$

The function $\phi$ is Borel and $\phi^{-1}(n)$ is $F_{W}$-independent for each $n$. We may therefore set $\mathcal{A}_{n}=\phi^{-1}(n)$. 
We now need a refinement of the notion of maximality for a cross section. Let $\mathcal{C} \subseteq X$ be a $U$-lacunary cross section. Given a set $Y \subseteq X$, we say that $\mathcal{C}$ is a maximal $U$-lacunary cross section in $Y$ if there are no $y \in Y \backslash \mathcal{C}$ for which the set $\mathcal{C} \cup\{y\}$ is $U$-lacunary. In other words, $\mathcal{C}$ cannot be enlarged by an element from $Y$ while keeping $U$-lacunarity.

LEMMA 2.2. Let $U$ and $V$ be compact symmetric neighborhoods of the identity in $G$, and let $\mathcal{C}$ be a $U$-lacunary cross section. Set $W=V \cdot U^{2} \cdot V$, and let $\mathcal{A} \subseteq \mathcal{C}$ be an $F_{W^{-}}$ independent Borel set. There exists a Borel cross section $\mathcal{D}$ such that $\mathcal{C} \subseteq \mathcal{D}$ and $\mathcal{D}$ is maximal $U$-lacunary in $V \cdot \mathcal{A}$.

Proof. Let $\left(f_{n}\right)$ be a countable family dense in $V$, and set

$$
\begin{aligned}
Y_{0} & =\left\{x \in \mathcal{A}: f_{0}(x) \notin U^{2} \cdot \mathcal{C}\right\}, \\
Y_{1} & =\left\{x \in \mathcal{A}: f_{1}(x) \notin U^{2} \cdot\left(\mathcal{C} \cup f_{0}\left(Y_{0}\right)\right)\right\}, \\
Y_{2} & =\left\{x \in \mathcal{A}: f_{2}(x) \notin U^{2} \cdot\left(\mathcal{C} \cup f_{0}\left(Y_{0}\right) \cup f_{1}\left(Y_{1}\right)\right)\right\}, \\
& \vdots \\
Y_{k} & =\left\{x \in \mathcal{A}: f_{k}(x) \notin U^{2} \cdot\left(\mathcal{C} \cup \bigcup_{i<k} f_{i}\left(Y_{i}\right)\right)\right\} .
\end{aligned}
$$

In words, $Y_{0}$ contains all $x \in \mathcal{A}$ such that $f_{0}(x)$ can be added to $\mathcal{C}$ while preserving $U$ lacunarity; $Y_{1}$ consists of those $x \in \mathcal{A}$ such that $f_{1}(x)$ can be added to $\mathcal{C} \cup f_{0}\left(Y_{0}\right)$ keeping $U$-lacunarity, etc.

Note that $\mathcal{C} \cup f_{0}\left(Y_{0}\right)$ is $U$-lacunary. Indeed, suppose $y, z \in \mathcal{C} \cup f_{0}\left(Y_{0}\right)$ are such that $g_{1} y=g_{2} z$ for some $g_{1}, g_{2} \in U$ and $y \neq z$. Since $\mathcal{C}$ is $U$-lacunary, at least one of $y, z$ has to be in $f_{0}\left(Y_{0}\right)$, say $z \in f_{0}\left(Y_{0}\right)$. If $y \in \mathcal{C}$, then $z=g_{2}^{-1} g_{1} y$ implies $z \in U^{2} \cdot \mathcal{C}$, which contradicts the construction of $Y_{0}$. Hence $y \in f_{0}\left(Y_{0}\right)$. Let $x_{y}, x_{z} \in Y_{0}$ be such that $y=$ $f_{0} x_{y}, z=f_{0} x_{z}$. Equality $z=g_{2}^{-1} g_{1} y$ implies $x_{z}=f_{0}^{-1} g_{2}^{-1} g_{1} f_{0}\left(x_{y}\right)$. Since

$$
f_{0}^{-1} g_{2}^{-1} g_{1} f_{0} \in V \cdot U^{2} \cdot V=W
$$

and $x_{y}, x_{z} \in \mathcal{A}$, the set $\mathcal{A}$ is not $F_{W}$-independent. This is a contradiction.

This shows that $\mathcal{C} \cup f_{0}\left(Y_{0}\right)$ is $U$-lacunary. Similarly, one checks that $\mathcal{C} \cup f_{0}\left(Y_{0}\right) \cup$ $f_{1}\left(Y_{1}\right)$ is $U$-lacunary and, in fact, $\mathcal{D}=\mathcal{C} \cup \bigcup_{i} f_{i}\left(Y_{i}\right)$ is $U$-lacunary. It remains to see that $\mathcal{D}$ is maximal $U$-lacunary in $V \cdot \mathcal{A}$.

To begin with, for any $n$ and any $x \in \mathcal{A}$ such that $f_{n}(x) \notin \mathcal{D}, \mathcal{D} \cup\left\{f_{n}(x)\right\}$ is not $U$ lacunary, for otherwise $\mathcal{C} \cup \bigcup_{i<n} f_{i}\left(Y_{i}\right) \cup\left\{f_{n}(x)\right\}$ would be $U$-lacunary, and hence $x \in$ $Y_{n}$, implying that $f_{n}(x) \in f_{n}\left(Y_{n}\right) \subseteq \mathcal{D}$. In other words, $\mathcal{D}$ cannot be enlarged to a $U$ lacunary cross section by adding an element of the form $f_{n}(x)$, for some $x \in \mathcal{A}$.

Suppose there is some $x \in \mathcal{A}$ and $y \in V \cdot x$ such that $y \notin \mathcal{D}$ and $\mathcal{D} \cup\{y\}$ is $U$-lacunary. Let $g \in V$ be such that $g x=y$ and pick $\left(n_{k}\right)_{k=0}^{\infty}$ for which $f_{n_{k}} \rightarrow g$. Since $U$ is a neighborhood of the identity, we may assume that $f_{n_{k}} g^{-1} \in U$ for all $k$; in particular $f_{n_{k}} x \notin \mathcal{D}$ for all $k$. But we showed that $\mathcal{D}$ cannot be enlarged to a $U$-lacunary cross section by an element of the form $f_{n_{k}} x$, and hence there are $z_{k} \in\left(U^{2} \cdot V \cdot x\right) \cap \mathcal{D}$ such that $h_{k} f_{n_{k}} x=z_{k}$ for some $h_{k} \in U^{2}$. Since $\left(U^{2} \cdot V \cdot x\right) \cap \mathcal{D}$ is finite, by passing to a subsequence, we may assume that $h_{k} f_{n_{k}} x=z$ for some fixed $z \in \mathcal{D}$ and all $k$. Recall that $U$ is compact, and so, by passing to a subsequence once again, we may assume that 
$h_{k} \rightarrow h \in U^{2}$. Let $p \in G$ be some element such that $p z=x$, for example, $p=\left(h_{0} f_{n_{0}}\right)^{-1}$. Thus $p h_{k} f_{n_{k}} x=x$ for all $k$ : i.e., elements $p h_{k} f_{n_{k}}$ are in the stabilizer of $x$. By Miller's theorem [Kec95, Theorem 9.17] stabilizers are closed, and thus phgx $=x$ : hence $h g x=p^{-1} x=z$. But $g x=y, h \in U^{2}$ and $z \in \mathcal{D}$, and hence $\mathcal{D} \cup\{y\}$ is not $U$-lacunary. This proves the claim and the lemma.

LEMMA 2.3. Let $U$ and $V$ be compact symmetric neighborhoods of the identity in $G$, and let $\mathcal{C}$ be a $U$-lacunary cross section. There exists a $U$-lacunary cross section $\mathcal{D}$ containing $\mathcal{C}$ which is maximal $U$-lacunary in $V \cdot \mathcal{C}$.

Proof. Let $W=V \cdot U^{2} \cdot V$. By Lemma 2.1, we may write $\mathcal{C}=\bigsqcup_{n} \mathcal{A}_{n}$ with each $\mathcal{A}_{n}$ being Borel and $F_{W}$-independent. Using Lemma 2.2, we construct inductively $U$-lacunary cross sections $\mathcal{C} \subseteq \mathcal{C}_{0} \subseteq \mathcal{C}_{1} \subseteq \cdots$ such that $\mathcal{C}_{i}$ is maximal $U$-lacunary in $V \cdot \mathcal{A}_{i}$. It is easy to see that $\mathcal{D}=\bigcup_{n} \mathcal{C}_{n}$ is maximal $U$-lacunary in $V \cdot \mathcal{C}$.

THEOREM 2.4. Let $U$ be a compact symmetric neighborhood of the identity in G. For every $U$-lacunary Borel cross section $\mathcal{C} \subseteq X$ there exists a maximal Borel $U$-lacunary cross section $\mathcal{D} \subseteq X$ such that $\mathcal{C} \subseteq \mathcal{D}$. The cross section $\mathcal{D}$ is therefore $U^{2}$-cocompact.

Proof. Fix an increasing sequence $\left(V_{n}\right)_{n=1}^{\infty}$ of symmetric compact neighborhoods of the identity which cover $G$ : i.e., $G=\bigcup_{n} V_{n}$. Applying Lemma 2.3, construct $U$-lacunary cross sections $\mathcal{C}=\mathcal{C}_{0} \subseteq \mathcal{C}_{1} \subseteq \mathcal{C}_{2} \subseteq \cdots$ such that $\mathcal{C}_{i}$ is maximal $U$-lacunary in $V_{i} \cdot \mathcal{C}_{i-1}$ (and, in particular, $\mathcal{C}_{i}$ is maximal $U$-lacunary in $V_{i} \cdot \mathcal{C}$ ). We claim that $\mathcal{D}=\bigcup_{n} \mathcal{C}_{n}$ is maximal $U$-lacunary: i.e., we claim that there are no $y \in X \backslash \mathcal{D}$ for which the cross section $\mathcal{D} \cup\{y\}$ is $U$-lacunary. Indeed, take $y \in X \backslash \mathcal{D}$ and let $x \in \mathcal{C}$ be such that $y \in G x$. Pick $n$ so large that $y \in V_{n} \cdot x$. Since $\mathcal{D}$ is maximal $U$-lacunary in $V_{n} \cdot \mathcal{C}, \mathcal{D} \cup\{y\}$ cannot be $U$-lacunary, and the claim follows.

\section{Orbit equivalences of locally compact group actions}

From now on we consider only free actions of groups.

Let $H$ be a locally compact group and let $\lambda$ be a right invariant Haar measure on $H$. Suppose also that $H$ acts freely on a standard Borel space $X$. Once we select a point $x \in X$, the orbit $\operatorname{Orb}(x)$ can be identified with the group $H$ itself via

$$
H \ni h \mapsto h x \in \operatorname{Orb}(x) .
$$

This identification makes it possible to transfer the measure $\lambda$ from $H$ onto the orbit $\operatorname{Orb}(x)$ by setting, for a set $A \subseteq \operatorname{Orb}(x)$,

$$
\lambda_{x}(A)=\lambda(\{h \in H: h x \in A\}) .
$$

The right invariance of $\lambda$ implies that $\lambda_{x}(A)=\lambda_{y}(A)$ whenever $y \in \operatorname{Orb}(x)$ : if $f x=y$, $f \in H$, then

$$
\begin{aligned}
\lambda_{y}(A) & =\lambda(\{h \in H: h y \in A\}) \\
& =\lambda(\{h \in H: h f x \in A\}) \\
& =[g:=h f] \\
& =\lambda\left(\{g \in H: g x \in A\} f^{-1}\right) \\
& =\lambda_{x}(A),
\end{aligned}
$$


and therefore the push forward of the Haar measure onto the orbit $\operatorname{Orb}(x)$ is independent of the base point. When $H$ is discrete, the measure on an orbit is just the counting measure, and any orbit equivalence thus automatically preserves the counting measure. This results in the fact that any orbit equivalence induces a bijection between the sets of invariant measures on the phase spaces. In the non-discrete world this is may no longer be the case. To fix this, we introduce a strengthening of $\mathrm{OE}$ which requires the isomorphism between phase spaces to preserve Haar measures on orbits. There are three increasingly restrictive rigorous formulations of this concept.

Let $G_{1} \curvearrowright X_{1}$ and $G_{2} \curvearrowright X_{2}$ be a pair of free Borel actions of locally compact groups, and pick right invariant Haar measures $\lambda_{1}$ and $\lambda_{2}$ on $G_{1}$ and $G_{2}$, respectively. We say that the actions $G_{1} \curvearrowright X_{1}$ and $G_{2} \curvearrowright X_{2}$ are weakly Haar orbit equivalent (abbreviated by wHOE) if there exists an orbit equivalence $\phi: X_{1} \rightarrow X_{2}$ such that, for any $x \in X_{1}$, the push forward measure $\phi_{*} \lambda_{1, x}$ is a multiple of $\lambda_{2, \phi(x)}$. In other words, the actions are wHOE if there exists an orbit equivalence which sends a Haar measure on each orbit onto a Haar measure. Note that we allow the normalization to vary from orbit to orbit. It is evident that the definition of wHOE does not depend on the choice of $\lambda_{1}$ and $\lambda_{2}$. Given a wHOE $\phi: X_{1} \rightarrow X_{2}$, we may associate a normalization function

$$
\alpha_{\phi}^{\lambda_{1}, \lambda_{2}}=\alpha_{\phi}: X_{1} \rightarrow \mathbb{R}^{>0} \quad \text { defined by the condition } \phi_{*} \lambda_{1, x}=\alpha_{\phi}(x) \lambda_{2, \phi(x)} .
$$

Note that $\alpha_{\phi}(x)=\alpha_{\phi}(y)$ whenever $x$ and $y$ are $E_{X_{1}}^{G_{1}}$-equivalent. The function $\alpha_{\phi}$ does depend on the choice of $\lambda_{1}$ and $\lambda_{2}$, but in a mild way: if $\alpha_{\phi}^{\lambda_{1}^{\prime}, \lambda_{2}^{\prime}}$ is defined with respect to some other choice of right Haar measures $\lambda_{1}^{\prime}$ and $\lambda_{2}^{\prime}$, then $\alpha_{\phi}^{\lambda_{1}^{\prime}, \lambda_{2}^{\prime}}$ is a constant multiple of $\alpha_{\phi}^{\lambda_{1}, \lambda_{2}}$. The property of $\alpha_{\phi}$ being constant is therefore independent of the choice of $\lambda_{1}$ and $\lambda_{2}$. This allows us to introduce the following definition.

Definition 3.1. Given a pair of free actions $G_{1} \curvearrowright X_{1}$ and $G_{2} \curvearrowright X_{2}$, we say that these actions are Haar orbit equivalent (HOE) whenever there exists a weak Haar orbit equivalence $\phi: X_{1} \rightarrow X_{2}$ with the constant normalization function: $\alpha_{\phi}^{\lambda_{1}, \lambda_{2}} \equiv$ const for some (equivalently, any) right Haar measures $\lambda_{1}, \lambda_{2}$ on $G_{1}$ and $G_{2}$, respectively.

One could reformulate HOE by saying that, given $\lambda_{1}$ for a suitable choice of $\lambda_{2}$, the push forward $\phi_{*} \lambda_{1, x}$ is equal to the measure $\lambda_{2, \phi(x)}$ for all $x \in X_{1}$.

Sometimes one has a natural normalization choice of the Haar measure, the Euclidean space $\mathbb{R}^{d}$ being a notable example. One may then wonder whether for a given choice of $\lambda_{1}$ and $\lambda_{2}$ on $G_{1}$ and $G_{2}$ there exists a $\operatorname{HOE} \phi: X_{1} \rightarrow X_{2}$ such that the normalization function is constantly equal to one. This brings us to our last and strongest definition.

Definition 3.2. Let $\lambda_{1}$ and $\lambda_{2}$ be right Haar measures on $G_{1}$ and $G_{2}$, and let $G_{1} \curvearrowright X_{1}$, $G_{2} \curvearrowright X_{2}$ be a pair of free actions. We say that these actions are Lebesgue orbit equivalent (LOE) if there exists a $\operatorname{HOE} \phi: X_{1} \rightarrow X_{2}$ such that the corresponding normalization function is constantly equal to 1: i.e., $\alpha_{\phi}^{\lambda_{1}, \lambda_{2}}(x)=1$ for all $x \in X_{1}$.

Equivalently, $\phi_{*} \lambda_{1, x}=\lambda_{1, \phi(x)}$ holds for all $x \in X_{1}$. Whereas wHOE and HOE do not depend on the choice of $\lambda_{1}$ and $\lambda_{2}$, the notion of LOE requires a choice of Haar measures. We shall always equip Euclidean spaces $\mathbb{R}^{d}$ with the standard Lebesgue measure, and LOE between $\mathbb{R}^{d}$-flows should always be understood with respect to that choice of Haar measure. 


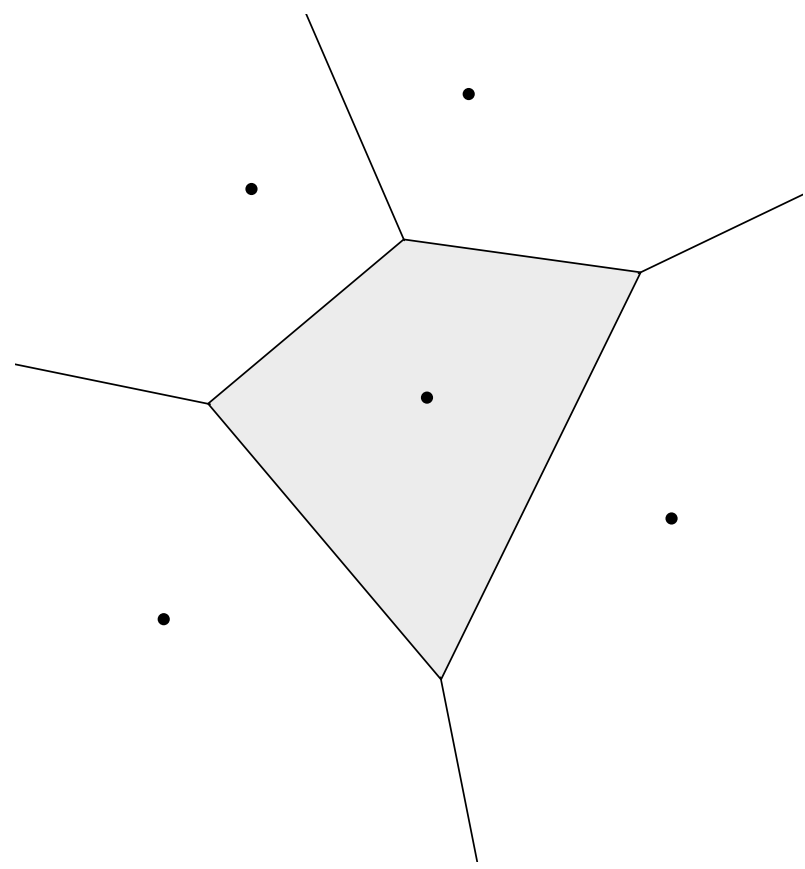

FIGURE 2. Voronoi tessellation.

\section{Invariant measures on cross sections and phase spaces}

In this section $G$ is assumed to be a unimodular $\dagger$ locally compact Polish group acting freely on $X$. We fix a Haar measure $\lambda$ and a compatible proper $\ddagger$ left invariant metric $d$ on $G$.

4.1. Voronoi domains. Let $E=E_{X}^{G}$ denote the orbit equivalence relation of the action: i.e., $(x, y) \in E$ whenever there exists some $g \in G$ such that $g x=y$. Note that freeness of the action implies uniqueness of such an element $g \in G$. The set $E$ is Borel and so is the function $\rho: E \rightarrow G$ that assigns to a pair $(x, y) \in E$ the unique $g \in G$ such that $g x=y$.

Given a cross section for the action $G \curvearrowright X$, we associate a decomposition of each orbit into Voronoi domains, which are determined by the proximity to the points in the cross section, as measured by the metric $d$. Let us first recall the construction in the more familiar setting of the Euclidean space followed by the formal definition of the general case.

Let $\mathcal{C} \subseteq \mathbb{R}^{d}$ be a cross section. A Voronoi domain of a point $c \in \mathcal{C}$ consists of all the points $x \in \mathbb{R}^{d}$ such that $x$ is closer to $c$ in the Euclidean distance than to any other point in $\mathcal{C}$. Voronoi tessellation is the partition of $\mathbb{R}^{d}$ into Voronoi domains. Figure 2 shows a

$\dagger$ A locally compact group is unimodular if its left Haar measure is also a right Haar measure, i.e., if the Haar measure is two-sided invariant.

\$ A compatible metric $d$ on a locally compact group is proper if all balls $B_{r}(e)=\{g \in G: d(g, e) \leq r\}$ are compact. Existence of such metrics has been established in [Str74]. 
fragment of Voronoi tessellation on $\mathbb{R}^{2}$ determined by the five points. The gray polygon is the Voronoi domain of the central point. Note that attribution of points on the boundary of domains is ambiguous.

In the general set-up of a unimodular group action, we start by fixing a Borel linear order $\preceq_{\mathcal{C}}$ on the cross section. It will be used to distribute the boundary points between the Voronoi domains. The Voronoi tiling determined by $\mathcal{C}$ (also known as Voronoi tessellation or Voronoi partition) is the set $\mathscr{V} \subseteq X \times \mathcal{C}$ given by

$$
\begin{gathered}
\mathscr{V}=\{(x, c) \in E: \forall a \in \mathcal{C} \cap(G \cdot c)(d(\rho(x, c), e)<d(\rho(x, a), e)) \text { or } \\
(d(\rho(x, c), e)=d(\rho(x, a), e) \text { and } c \preceq \mathcal{C} a)\} .
\end{gathered}
$$

In words, $(x, c) \in \mathscr{V}$ if $c$ is closer to $x$ than any other point in $\mathcal{C}$, or, when there are several closest points in $\mathcal{C}, c$ is the minimal one according to $\preceq \mathcal{C}$. Note that, by lacunarity, there can only be a finite number of closest points, so the minimal one always exists. The Voronoi domain of $c \in \mathcal{C}$ is the set

$$
\mathscr{V}_{c}=\{x \in X:(x, c) \in \mathscr{V}\} .
$$

Note that if $\mathcal{C}$ is cocompact, then Voronoi domains $\mathscr{V}_{c}$ are bounded (in the sense that $d(\rho(\cdot, c), e): \mathscr{V}_{c} \rightarrow \mathbb{R}$ is bounded) uniformly in $c$.

The set $\mathscr{V}$ is an example of a tiling of the action $G \curvearrowright X$.

Definition 4.1. A tiling of $G \curvearrowright X$ is a Borel set $\mathscr{W} \subseteq X \times X$ satisfying the following properties.

(i) Projection $\mathcal{C}$ of the set $\mathscr{W}$ onto the second coordinate is Borel and forms a cross section of the action,

$$
\mathcal{C}=\{c \in X:(x, c) \in X \text { for some } x \in X\} .
$$

We refer to this cross section as the one associated with $\mathscr{W}$. By definition $\mathscr{W} \subseteq$ $X \times \mathcal{C}$.

(ii) $\mathscr{W} \subseteq E_{X}^{G}$ : i.e., $\mathscr{W}$ respects the orbit equivalence relation.

(iii) For any $x \in X$ there exists a unique $c \in \mathcal{C}$ with $(x, c) \in \mathscr{W}$ : i.e., $\mathscr{W}$ is a graph of a Borel function $w: X \rightarrow \mathcal{C}$.

(iv) There exists a neighborhood of the identity $U \subseteq G$ such that $\mathcal{C}$ is $U$-lacunary and $U c \times\{c\} \subseteq \mathscr{W}$ for all $c \in \mathcal{C}$.

Given a tiling $\mathscr{W}$, the domain of a point $c \in \mathcal{C}$ is the set $\mathscr{W}_{c}$ of all $x \in X$ such that $(x, c) \in \mathscr{W}$. Geometrically, any orbit $\mathcal{O} \subseteq X$ is therefore tiled by sets $\mathscr{W}_{c}, c \in \mathcal{O} \cap \mathcal{C}$.

We say that a tiling is bounded if the cross section $\mathcal{C}$ associated with $\mathscr{W}$ is cocompact and domains $\mathscr{W}_{c}$ are bounded uniformly in $c$, in the sense that there exists a compact set $V \subseteq G$ such that $\mathscr{W}_{c} \subseteq V \cdot c$ for all $c \in \mathcal{C}$.

Note that, given any cocompact cross section $\mathcal{C} \subseteq X$, the Voronoi tessellation constructed above is an example of a bounded tiling of $G \curvearrowright X$ with associated cross section $\mathcal{C}$.

Our goal in the next few subsections is to establish a correspondence between finite invariant measures on the phase space $X$ and on a cocompact cross section $\mathcal{C}$. 
4.2. Lifting measures to the phase space. Let $v$ be a finite invariant measure on a cocompact cross section $\mathcal{C}$. We are going to lift $v$ to a finite invariant measure $\mu_{v}$ on $X$. For this, we fix a bounded tiling $\mathscr{W}$ of $G \curvearrowright X$ for which the associated cross section is $\mathcal{C}$ : e.g., the Voronoi tessellation constructed above. We mention, in advance, that the measure $\mu_{v}$ will turn out to be independent of the choice of $\mathscr{W}$. Given a Borel set $A \subseteq X$ and $c \in \mathcal{C}$, let $\xi(A, c)$ be defined by

$$
\xi(A, c)=\lambda\left(\left\{g \in G: g c \in \mathscr{W}_{c} \cap A\right\}\right) .
$$

For a fixed $c \in \mathcal{C}, \xi(\cdot, c)$ is a finite (albeit not invariant) measure on $X$. Measures $\xi(\cdot, c)$ are bounded uniformly in $c$ since $\mathscr{W}$ is assumed to be bounded. The measure $\mu_{v}$ is then defined to be the integration of $\xi(\cdot, c)$ with respect to $v$ : i.e., for a Borel $A \subseteq X$

$$
\mu_{\nu}(A)=\int_{\mathcal{C}} \xi(A, c) d v(c) .
$$

We would like to show that $\mu_{v}$ is $G$-invariant. Let $T: X \rightarrow X$ be a Borel automorphism of the orbit equivalence relation $E_{X}^{G}$, and suppose that $T$ preserves the Haar measure on each orbit: for any orbit $\mathcal{O} \subseteq X$, any Borel set $P \subseteq \mathcal{O}$ and any (equivalently, some) $x \in \mathcal{O}$ one has $\lambda_{x}(P)=\lambda_{x}(T P)$, or in a more detailed form,

$$
\lambda(\{g \in G: g x \in P\})=\lambda(\{g \in G: g x \in T P\}) .
$$

Proposition 4.2. In the notation above, $\mu_{v}(T A)=\mu_{v}(A)$ for all Borel $A \subseteq X$.

Proof. Let $H$ be a countable group, with an enumeration of its element $\left(h_{n}\right)_{n=1}^{\infty}$, such that the relation

$$
E_{\mathcal{C}}=E_{X}^{G} \cap(\mathcal{C} \times \mathcal{C})
$$

is given by an action $H \curvearrowright \mathcal{C}$ (existence of such an action is guaranteed by a theorem of Feldman and Moore [FM77]). Fix a Borel set $A \subseteq X$ and let

$$
A_{n}^{\prime}=\left\{x \in A: h_{n} w(x)=w(T x)\right\},
$$

where $w: X \rightarrow \mathcal{C}$ is a function which has graph $\mathscr{W}$. The sets $A_{n}^{\prime}$ may not be disjoint, so we also define $A_{1}=A_{1}^{\prime}$ and $A_{n}=A_{n}^{\prime} \backslash \bigcup_{k<n} A_{k}$. This gives us a partition $A=\bigsqcup_{n} A_{n}$. Since

$$
\mu_{v}(A)=\sum_{n} \mu_{v}\left(A_{n}\right) \quad \text { and } \quad \mu_{v}(T A)=\sum_{n} \mu_{v}\left(T A_{n}\right),
$$

to prove the proposition it is enough to show that $\mu_{v}\left(T A_{n}\right)=\mu_{v}\left(A_{n}\right)$ for each $n$.

Sets $A_{n}$ are constructed in such a way that, for any $c \in \mathcal{C}$,

$$
T\left(A_{n} \cap \mathscr{W}_{c}\right)=T A_{n} \cap \mathscr{W}_{h_{n} c},
$$

and therefore the assumption that $T$ preserves the Haar measure within each orbit implies

$$
\xi\left(A_{n}, c\right)=\xi\left(T A_{n}, h_{n} c\right) \quad \text { for all } c \in \mathcal{C} .
$$


We therefore conclude that

$$
\begin{aligned}
\mu_{v}\left(T A_{n}\right) & =\int_{\mathcal{C}} \xi\left(T A_{n}, c\right) d v(c) \\
& =\left[\tilde{c}:=h_{n}^{-1} c\right] \\
& =\int_{\mathcal{C}} \xi\left(T A_{n}, h_{n} \tilde{c}\right) d v\left(h_{n} \tilde{c}\right) \\
& =\int_{\mathcal{C}} \xi\left(A_{n}, \tilde{c}\right) d v(\tilde{c}) \\
& =\mu_{v}\left(A_{n}\right),
\end{aligned}
$$

where the penultimate equality follows from (2) and from the $H$-invariance of $\nu$.

COROLlaRY 4.3. The measure $\mu_{v}$ is G-invariant.

Proof. For $g \in G$ set $T_{g}(A)=g A$ and apply Proposition 4.2.

Note that the construction of $\mu_{v}$ and Proposition 4.2 are valid when $G$ is not necessarily unimodular and $\lambda$ is merely a right invariant Haar measure, but Corollary 4.3 uses left invariance of $\lambda$.

For future reference, we note that, if a neighborhood of the identity $U \subseteq G$ is such that $U \times\{c\} \subseteq \mathscr{W}$ for all $c \in \mathcal{C}$, then the map $U \times \mathcal{C} \ni(g, c) \mapsto g c \in U \cdot \mathcal{C}$ is a bijection and, via this identification,

$$
\left.\mu_{v}\right|_{U \cdot \mathcal{C}}=\left.\lambda\right|_{U} \times \nu
$$

4.3. Pulling measures to a cross section. One can pull measures in the opposite direction as well. Pick $U \subseteq G$ to be such a small neighborhood of the identity that $\mathcal{C}$ is $U$-lacunary. If $\mu$ is an invariant measure on the phase space $X$, we set, for $\mathcal{A} \subseteq \mathcal{C}$, the measure $v_{\mu}(\mathcal{A})$ to be defined by

$$
v_{\mu}(\mathcal{A})=\frac{\mu(U \cdot \mathcal{A})}{\lambda(U)} .
$$

The definition turns out to be independent of the choice of $U$. The measure $v_{\mu}$ is a finite invariant measure on $\mathcal{C}$ and, moreover,

$$
\left.\mu\right|_{U \cdot \mathcal{C}}=\left.\lambda\right|_{U} \times v_{\mu} .
$$

For a slick proof of these assertions see [KPV13, Proposition 4.3]. Whereas cocompactness of $\mathcal{C}$ was used in $\S 4.2$ to ensure that $\mu_{v}$ is finite, results of this subsection are valid even when $\mathcal{C}$ is not necessarily cocompact.

4.4. Correspondence between ergodic measures. The maps $\mu \mapsto v_{\mu}$ and $\nu \mapsto \mu_{v}$ are inverses of each other. Indeed, (3) and (4) imply that $v_{\mu_{v}}$ is such that, via the natural identifications,

$$
\left.\lambda\right|_{U} \times v_{\mu_{v}}=\left.\mu_{v}\right|_{U} \cdot \mathcal{C}=\left.\lambda\right|_{U} \times v,
$$

and therefore $v_{\mu_{v}}=v$ for any finite invariant measure $v$ on $\mathcal{C}$. On the other hand, for any invariant measure $\mu$ on $X$

$$
\left.\mu_{v_{\mu}}\right|_{U \cdot \mathcal{C}}=\left.\lambda\right|_{U} \times v_{\mu}=\left.\mu\right|_{U \cdot \mathcal{C}},
$$


implying that $\left.\mu_{v_{\mu}}\right|_{U \cdot \mathcal{C}}=\left.\mu\right|_{U \cdot \mathcal{C}}$. But both measures are $G$-invariant and hence $\mu_{v_{\mu}}=\mu$. In particular, the construction of $\mu_{\nu}$ does not depend on the choice of a bounded tiling $\mathscr{W}$.

While the map $\mu \mapsto v_{\mu}$ is a linear bijection between spaces of invariant measures, it does not preserve the normalization: in general, $\mu(X) \neq v_{\mu}(\mathcal{C})$. Let $\mathcal{E}(X)$ denote the family of all pie measures on $X$ (recall that pie stands for probability invariant ergodic); invariance and ergodicity is understood to be with respect to the orbit equivalence relations $E_{X}^{G}$. Similarly let $\mathcal{E}(\mathcal{C})$ denote the family of pie measures on $\mathcal{C}$ with respect to the relation $E_{\mathcal{C}}$.

Proposition 4.4. The map $\mathcal{E}(X) \ni \mu \mapsto v_{\mu} / v_{\mu}(\mathcal{C}) \in \mathcal{E}(\mathcal{C})$ is a bijection between $\mathcal{E}(X)$ and $\mathcal{E}(\mathcal{C})$.

Proof. We start by checking that, for any finite invariant measure $\mu$ on $X$, the measure $v_{\mu}$ is ergodic if and only if $\mu$ is ergodic. Indeed, suppose $v_{\mu}$ is ergodic and let $Z \subseteq X$ be a $G$-invariant set. Since $Z \cap \mathcal{C}$ is $E_{\mathcal{C}}$-invariant, either $v_{\mu}(Z \cap \mathcal{C})=0$ or $v_{\mu}(\mathcal{C} \backslash Z)=0$. Suppose, for definiteness, that the former is realized. By (4), $\mu(U \cdot(Z \cap \mathcal{C}))=0$, and therefore $\mu(Z)=0$. Thus $\mu$ must be ergodic.

If $\mu$ is ergodic and $Z \subseteq \mathcal{C}$ is $E_{\mathcal{C}}$-invariant, then either $\mu(G \cdot Z)=0$ or $\mu(G \cdot(\mathcal{C} \backslash Z))=$ 0 . Equation (4) implies that either $v_{\mu}(Z)=0$ or $v_{\mu}(\mathcal{C} \backslash Z)=0$ must take place.

This proves that, for any $\mu \in \mathcal{E}(X)$, the measure $v_{\mu} / v_{\mu}(\mathcal{C})$ is indeed an element of $\mathcal{E}(\mathcal{C})$. The map $\mu \mapsto v_{\mu} / v_{\mu}(\mathcal{C})$ is injective since, if $\mu_{1} \neq \mu_{2}$ are pie measure on $X$, there exists an invariant $Z \subseteq X$ such that $\mu_{1}(Z)=1$ and $\mu_{2}(Z)=0$. This implies that $v_{\mu_{1}}(Z \cap \mathcal{C})>0$ and $v_{\mu_{2}}(Z \cap \mathcal{C})=0$ : therefore $v_{\mu_{1}} / v_{\mu_{1}}(\mathcal{C})$ and $v_{\mu_{2}} / v_{\mu_{2}}(\mathcal{C})$ are distinct.

Finally, the map is surjective. Indeed, for a given $v \in \mathcal{E}(\mathcal{C})$ there exists a finite invariant measure $\mu$ on $X$ such that $v_{\mu}=v$. By the above, the measure $\mu$ must be ergodic, and hence $\mu / \mu(X) \in \mathcal{E}(X)$. But also

$$
v_{a \mu}=a v_{\mu} \quad \text { for all } a \in \mathbb{R}^{>0},
$$

and therefore

$$
\frac{v_{\mu / \mu(X)}}{v_{\mu / \mu(X)}(\mathcal{C})}=\frac{v_{\mu}}{v_{\mu}(\mathcal{C})}=v .
$$

We conclude this section with an observation that wHOE induces a bijection between pie measures on the phase spaces.

THEOREM 4.5. Let $G_{1} \curvearrowright X_{1}$ and $G_{2} \curvearrowright X_{2}$ be free Borel actions of unimodular locally compact Polish groups $G_{1}$ and $G_{2}$, and let $\phi: X_{1} \rightarrow X_{2}$ be a wHOE between the actions. The push-forward map $\phi_{*}: \mathcal{E}\left(X_{1}\right) \rightarrow \mathcal{E}\left(X_{2}\right)$ induces a bijection between pie measures on $X_{1}$ and $X_{2}$.

Proof. The only thing that is not immediate from the definitions is that $\phi_{*} \mu$ is $G_{2}$-invariant whenever $\mu$ is a $G_{1}$-invariant measure. Let $Z \subseteq X_{2}$ and let $h \in G_{2}$. We need to show that $\phi_{*} \mu(Z)=\phi_{*} \mu(h Z)$, or in other words,

$$
\mu\left(\phi^{-1}(Z)\right)=\mu\left(\phi^{-1}(h Z)\right) .
$$

Let $T: X_{1} \rightarrow X_{1}$ be defined by $T x=\phi^{-1} \circ h \circ \phi(x)$. The map $T$ is a Borel bijection preserving $E_{X_{1}}^{G_{1}}$ and, moreover, we claim that $T$ preserves the Haar measure on each orbit 
of $G_{1}$, therefore satisfying the assumptions of Proposition 4.2. Once this claim is proved, we get

$$
\mu\left(\phi^{-1}(Z)\right)=\mu\left(T \phi^{-1}(Z)\right)=\mu\left(\phi^{-1}(h Z)\right) .
$$

To see that $T$ preserves the Haar measure, let us pick an orbit $\mathcal{O} \subseteq X_{1}$ and a point $x_{1} \in \mathcal{O}$, and let $\lambda_{1}$ and $\lambda_{2}$ be Haar measures on $G_{1}$ and $G_{2}$, respectively. By the definition of wHOE, $\phi_{*} \lambda_{1, x}=\alpha_{\phi}(x) \lambda_{2, \phi(x)}$ for some $\alpha_{\phi}(x) \in \mathbb{R}^{>0}$ which is, moreover, constant on $\mathcal{O}$. For a subset $P \subseteq \mathcal{O}$, using that $\lambda_{2, \phi(x)}$ is $G_{2}$-invariant,

$$
\begin{aligned}
\lambda_{1, x}(T P) & =\lambda_{1, x}\left(\phi^{-1} \circ h \circ \phi(P)\right) \\
& =\alpha_{\phi}(x) \lambda_{2, \phi(x)}(h \circ \phi(P)) \\
& =\alpha_{\phi}(x) \lambda_{2, \phi(x)}(\phi(P)) \\
& =\lambda_{1, x}(P) .
\end{aligned}
$$

Thus $\lambda_{1, x}(T P)=\lambda_{1, x}(P)$ for all $P \subseteq \mathcal{O}$, and the theorem follows.

\section{Rectangular tilings of multidimensional flows}

From this section onward, we restrict ourselves to the case of a free Borel action $\mathbb{R}^{d} \curvearrowright X$ of the Euclidean space on a standard Borel space $X$. Recall that we use an additive notation for the action: for $x \in X$ and $r \in \mathbb{R}^{d}$ the action of $r$ upon $x$ is denoted by $x+r$.

Our main tool in understanding multidimensional flows is the concept of a rectangular tiling. Simply put, it is a Borel partition of orbits into rectangles $\dagger$.

Definition 5.1. A rectangular tiling of an action $\mathbb{R}^{d} \curvearrowright X$ is a tiling $\mathscr{R}$ (in the sense of Definition 4.1) such that, for the associated cross section $\mathcal{C}$ and any $c \in \mathcal{C}$, the domain $\mathscr{R}_{c}$ is a (half-open) rectangle: $\mathscr{R}_{c}=c+R_{c}$, where $R_{c}$ is of the form

$$
R_{c}=\prod_{i=1}^{d}\left[a_{i}, b_{i}\right) .
$$

Equivalently, a rectangular tiling is a cross section $\mathcal{C} \subseteq X$ together with bounded away from zero Borel functions (we call them dimension functions) $\zeta_{i}^{l}, \zeta_{i}^{r}: \mathcal{C} \rightarrow \mathbb{R}^{>0}, i \leq d$, such that rectangles $R_{c}=\prod_{i=1}^{d}\left[-\zeta_{i}^{l}(c), \zeta_{i}^{r}(c)\right)$ tile all the orbits: for any orbit $\mathcal{O} \subseteq X$,

$$
\mathcal{O}=\bigsqcup_{c \in \mathcal{C} \cap \mathcal{O}}\left(c+R_{c}\right) .
$$

Figure 3 provides an illustration of a rectangular tiling. The gray rectangle corresponds to a single tile of the form $c+R_{c}$. All our rectangles are half-open to ensure that $c+R_{c}$ and $c^{\prime}+R_{c^{\prime}}$ are disjoint whenever $c \neq c^{\prime}$. Note also that, given a tiling $\mathscr{R}$, we may select in a Borel way centers of tiles $c+\vec{w}_{c}$, where $\vec{w}_{c}(i)=\left(\zeta_{i}^{r}(c)-\zeta_{i}^{l}(c)\right) / 2$. Unless stated otherwise, we shall therefore assume that our tilings are symmetric and points $c \in \mathcal{C}$ are centers of the tiles $R_{c}=\prod_{i=1}^{d}\left[-\zeta_{i}(c), \zeta_{i}(c)\right)$.

Existence of rectangular tilings for actions of $\mathbb{Z}^{d}$ has been established by Gao and Jackson [GJ15]. They proved that any free action $\mathbb{Z}^{d} \curvearrowright X$ admits a rectangular tiling

$\dagger$ Perhaps it would be more accurate to speak of cuboids rather than rectangles, but since our figures illustrate the case $d=2$, and since the argument is the same in all dimensions, we choose to use the two-dimensional terminology throughout the paper. 


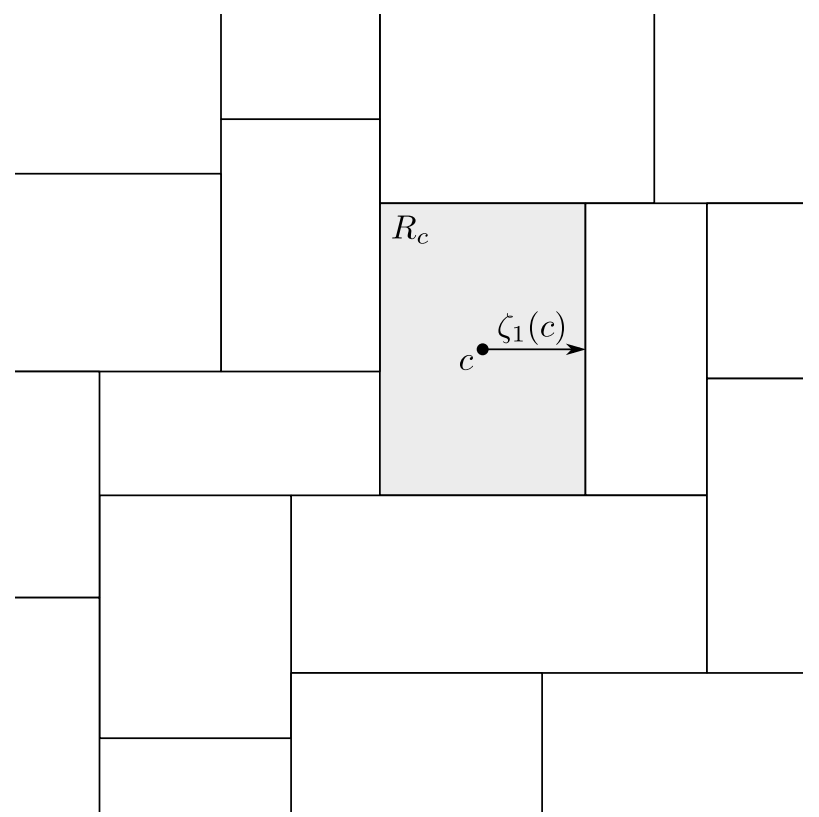

FIGURE 3. Rectangular tiling.

and, moreover, for any $L \in \mathbb{R}$ one may always find a tiling $\mathscr{R}$ such that the dimension functions are bounded below by $L$. A similar result is true for $\mathbb{R}^{d}$-flows.

THEOREM 5.2. (Gao-Jackson for $\mathbb{Z}^{d}$ actions) For any $L>0$ there exists a rectangular tiling of $\mathbb{R}^{d} \curvearrowright X$ with all edges of rectangles at least $2 L: \zeta_{i}(c) \geq L$ for all $i \leq d$ and all $c$ in the associated cross section $\mathcal{C}$.

What matters for the argument in [GJ15, §3] is the large-scale geometry of $\mathbb{Z}^{d}$, which is the same as that of $\mathbb{R}^{d}$; thus only superficial modifications for their proof are required, which we therefore omit.

Following [GJ15, Lemma 3.2], we may improve the above statement by imposing further restrictions on the dimension functions.

THEOREM 5.3. Let $\mathbb{R}^{d} \curvearrowright X$ be a free multidimensional flow. For any $L^{\prime}>0$ and any $\epsilon>0$ there exists a rectangular tiling $\mathscr{Q}$ of the flow such that

$$
\left|\zeta_{i}(c)-L^{\prime}\right|<\epsilon
$$

for all $c$ in the associated cross section $\mathcal{C}$.

Proof. Pick $L$ so large that any real $r \geq L$ can be partitioned into reals $\epsilon$-close to $L$ : for any $r \geq L$ there exist $s_{1}, \ldots, s_{n}>0$ such that $r=\sum_{i=1}^{n} s_{i}$ and $\left|s_{i}-L^{\prime}\right|<\epsilon$ for all $i \leq n$. Use Theorem 5.2 and construct a tiling $\mathscr{R}$ with all edges of tiles being at least $L$. By the choice of $L$, each tile $\mathscr{R}_{c}$ can be partitioned into rectangles $Q_{c, 1}, \ldots, Q_{c, n}$ having all edges $\epsilon$-close to $L^{\prime}$. These rectangles $Q_{c, i}$ constitute tiles of the desired tiling $\mathscr{Q}$. 


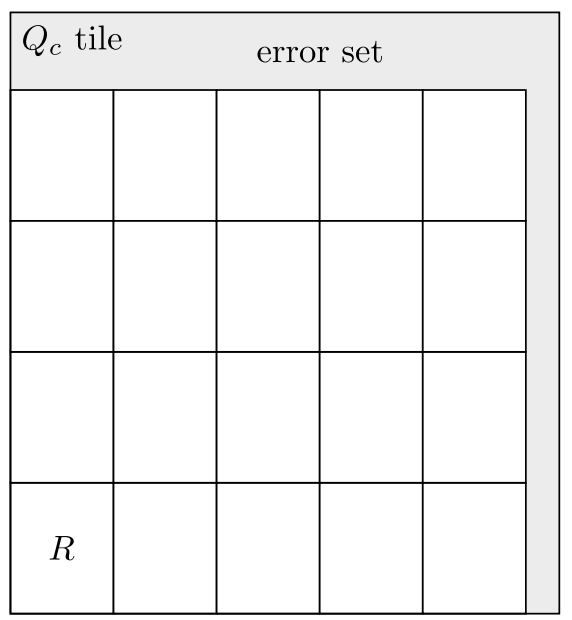

FIGURE 4. Large marker-small marker.

\section{Uniform Rokhlin's lemma}

The following theorem is the usual Rokhlin's lemma [Lin75, Theorem 1] when the measure $\mu$ is fixed. The adjective 'uniform' refers to the fact that $\mu(\mathcal{C}+R)>1-\epsilon$ holds for all invariant probability measures.

THEOREM 6.1. (Uniform Rokhlin's lemma for $\mathbb{R}^{d}$ actions) Let $\mathbb{R}^{d} \curvearrowright X$ be a free Borel action. For any rectangle $R=\prod_{i=1}^{d}\left[-a_{i}, a_{i}\right), a_{i}>0$ and any $\epsilon>0$, there exists a Borel $R$-lacunary cocompact cross section $\mathcal{C} \subseteq X$ such that $\mu(\mathcal{C}+R)>1-\epsilon$ for any invariant probability measure $\mu$ on $X$.

Proof. We begin with an application of Theorem 5.3 and select a tiling $\mathscr{Q}$ with associated cross section $\mathcal{C}_{0} \subseteq X$ and domains $\mathscr{Q}_{c}=c+Q_{c}$, such that each rectangle $Q_{c}$ is 'large' compared to $R$.

Now try to tile each $c+Q_{c}$ with copies of $R$ starting from the 'bottom left' corner as shown in Figure 4. Since lengths of edges of $Q_{c}$ are not necessarily multiples of the corresponding edges of $R$, there will be some remainder, also known as the error set, marked gray in Figure 4. The condition on the size of $Q_{c}$ is that the proportion of the measure of the error set to the measure of $\mathscr{Q}_{c}$ is at most $\epsilon$ : in the notation of $\S 4.2$,

$$
\xi(\text { error set, } c) / \xi\left(\mathscr{Q}_{c}, c\right)<\epsilon \text {. }
$$

Let $\mathcal{C}$ denote the cross section which consists of centers of all the rectangles $R$ inscribed into tiles $\mathscr{Q}_{c}$. We claim that $\mathcal{C}$ satisfies the desired properties.

The only thing that requires checking is that $\mu(\mathcal{C}+R)>1-\epsilon$ for all probability $E_{X^{-}}$ invariant measures $\mu$ on $X$. By $\S 4$, one can find a finite invariant measure $v$ on $\mathcal{C}_{0}$ such that

$$
\mu=\int_{\mathcal{C}} \xi(\cdot, c) d \nu(c) \quad \text { where } \xi(\cdot, c) \text { is the 'Lebesgue' measure on } \mathscr{Q}_{c} .
$$


By the construction of $\mathcal{C}$, one has $\xi(\mathcal{C}+R, c)>(1-\epsilon) \xi\left(\mathscr{Q}_{c}, c\right)=(1-\epsilon) \xi(X, c)$ and therefore

$$
\begin{aligned}
\mu(\mathcal{C}+R) & =\int_{\mathcal{C}_{0}} \xi(\mathcal{C}+R, c) d \nu(c)>\int_{\mathcal{C}_{0}}(1-\epsilon) \xi(X, c) d \nu(c) \\
& =(1-\epsilon) \mu(X) \\
& =1-\epsilon .
\end{aligned}
$$

For the proof of the main technical result, namely Theorem 6.3 below, we shall need an easy lemma. Suppose we have a square $R=\prod_{i=1}^{d}[-l, l)$ and an $R$-lacunary cross section $\mathcal{C}$. If we shrink $R$ to a square $R^{\leftarrow b}=\prod_{i=1}^{d}[-l+b, l-b)$, where $b$ is small compared to $l$, then $\mu\left(\mathcal{C}+R^{\leftarrow b}\right)$ has to be close to $\mu(\mathcal{C}+R)$ for all invariant measures $\mu$ on $X$.

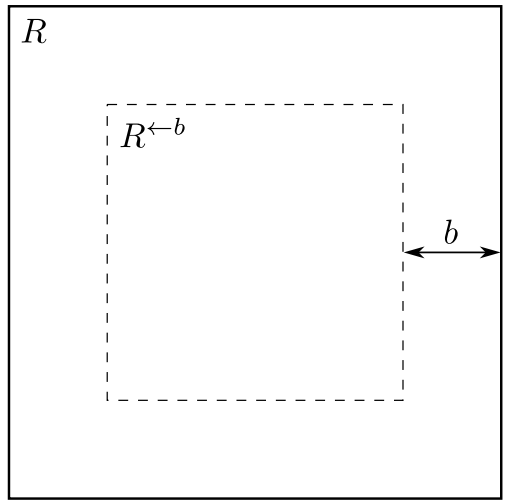

FIGURE 5. Shrinking $R$ to $R^{\leftarrow b}$.

In general, for any rectangle $R=\prod_{i=1}^{d}\left[l_{i}, r_{i}\right)$ and $b \in \mathbb{R}^{\geq 0}$, we let $R^{\leftarrow b}$ denote the rectangle $\prod_{i=1}^{d}\left[l_{i}+b, r_{i}-b\right)$. This notation will only be used when $l_{i}+b$ is still less than $r_{i}-b$. In that case $R^{\leftarrow b}$ is obtained by shrinking each edge of $R$ by $b$ (see Figure 5). Note that if $\widetilde{R}$ is any rectangle contained in $\prod_{i=1}^{d}[-L, L]$, then

$$
R^{\leftarrow b}+\widetilde{R} \subseteq R^{\leftarrow b-L} .
$$

LEMMA 6.2. Let $\mathbb{R}^{d} \curvearrowright X$ be a free Borel flow. For any $\epsilon>0$ and any real $b \in \mathbb{R}^{\geq 0}$, there exists $L \geq b$ such that, for any $l \geq L$, square $R=[-l, l)^{d}$, any $R$-lacunary cocompact cross section $\mathcal{C} \subseteq X$ and any probability invariant measure $\mu$ on $X$,

$$
\mu\left(\mathcal{C}+R^{\leftarrow b}\right)>\mu(\mathcal{C}+R)-\epsilon .
$$

Proof. Pick $L$ so large that $\lambda\left(R^{\leftarrow b}\right) / \lambda(R)>1-\epsilon$ for any $R=[-l, l)^{d}, l \geq L$, where $\lambda$ is the Lebesgue measure on $\mathbb{R}^{d}$. We claim that such $L$ works. Let $\mu$ be a probability invariant measure on $X$. Since $\mathcal{C}+R$ may be a proper subset of $X$, it does not form a tiling of the action. It is, nevertheless, easy to enlarge it into a tiling as follows. Let $\mathscr{V}$ be the Voronoi tiling determined by $\mathcal{C}$. Define $\mathscr{W}$ to be

$$
\mathscr{W}=\left\{(x, c):(x \in c+R) \text { or }\left((x, c) \in \mathscr{V} \text { and }\left((x, c) \notin c^{\prime}+R \text { for any } c^{\prime} \in \mathcal{C}\right)\right)\right\} .
$$


It is easy to see that $\mathscr{W}$ is a bounded tiling of $\mathbb{R}^{d} \curvearrowright X$ and we may therefore apply results of $\S 4$ to decompose $\mu$ as an integral over some measure $v$ on $\mathcal{C}$ of 'Lebesgue' measures $\xi(\cdot, c)$ : that is,

$$
\mu=\int_{\mathcal{C}} \xi(\cdot, c) d v(c)
$$

Since $\lambda\left(R^{\leftarrow b}\right)>(1-\epsilon) \lambda(R)$,

$$
\begin{aligned}
\mu\left(\mathcal{C}+R^{\leftarrow b}\right) & =\int_{\mathcal{C}} \xi\left(\mathcal{C}+R^{\leftarrow b}, c\right) d \nu(c) \\
& >(1-\epsilon) \int_{\mathcal{C}} \xi(\mathcal{C}+R, c) d \nu(c) \\
& =(1-\epsilon) \mu(\mathcal{C}+R) \\
& \geq \mu(\mathcal{C}+R)-\epsilon \mu(X) .
\end{aligned}
$$

Since the measure $\mu$ is assumed to be a probability measure, $\mu(X)=1$ and $\mu\left(\mathcal{C}+R^{\leftarrow b}\right)>$ $\mu(\mathcal{C}+R)-\epsilon$.

In ergodic theory, Rokhlin's lemma is frequently applied a countable number of times to build a cover of the phase space with a sequence of refining Rokhlin towers. Exact details vary from application to application, and the following theorem provides the set-up that will be needed in our case.

THEOREM 6.3. For any increasing sequence $\left(b_{n}\right)_{n=1}^{\infty}$ and any real $\kappa>0$, there exist an invariant Borel $Z \subseteq X$, an increasing sequence of reals $\left(l_{n}\right)_{n=1}^{\infty}$ and a sequence of Borel sets $\mathcal{C}_{n} \subseteq Z$ such that, for $R_{n}=\left[-l_{n}, l_{n}\right)^{d}$ :

(i) $\quad\left(c+R_{n+1}\right) \cap \mathcal{C}_{n} \neq \varnothing$ for each $c \in \mathcal{C}_{n+1}$;

(ii) $Z=\bigcup_{n}\left(\mathcal{C}_{n}+R_{n}\right)$;

(iii) each $l_{n}$ is an integer multiple of $\kappa$;

(iv) $l_{n} \geq b_{n}$;

(v) $\mathcal{C}_{n}$ is $R_{n}$-lacunary;

(vi) $\mathcal{C}_{n}+R_{n} \subseteq \mathcal{C}_{n+1}+R_{n+1}^{\leftarrow b_{n+1}}$; and

(vii) $\mu(Z)=1$ for any probability invariant measure $\mu$ on $X$.

Item (vi) is the most important one here. It says that each rectangle in $\mathcal{C}_{n}+R_{n}$ is inside a rectangle from $\mathcal{C}_{n+1}+R_{n+1}$ and, moreover, it is far from its boundary. Figure 6 gives an illustration of this item. While it would be convenient to have such a covering on all orbits, this is not always possible, and item (vii) offers the next best thing instead. Reasons for taking $l_{n}$ to be a multiple of $\kappa$ will be apparent in the proof of Theorem 7.1 , but this restriction is not essential. Note, also, that items (i) and (ii) imply that each $\mathcal{C}_{n}$ is a cross section of $\mathbb{R}^{d} \curvearrowright Z$.

Proof. Without loss of generality, we assume that $\lim _{n \rightarrow \infty} b_{n}=\infty$. Pick a decreasing sequence $\left(\epsilon_{n}\right)_{n=1}^{\infty}, \epsilon_{n}>0$, such that $\sum_{n} \epsilon_{n}<1$. Using Theorem 6.1 and Lemma 6.2 at each step, we construct inductively reals $\tilde{b}_{n}, l_{n}$ and $R_{n}$-lacunary cross sections $\mathcal{C}_{n}^{\prime} \subseteq X$, where $R_{n}=\left[-l_{n}, l_{n}\right)^{d}$, such that: 

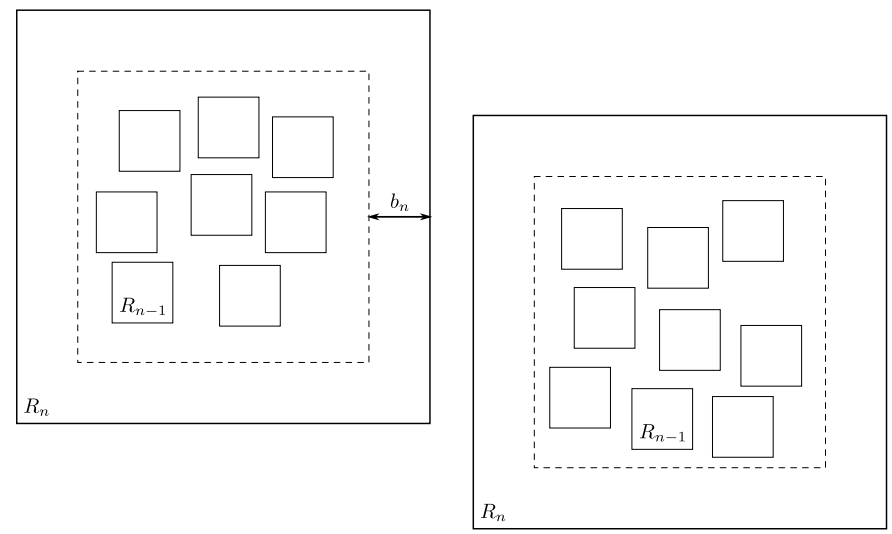

$R_{n+1}$

FIGURE 6. Rokhlin towers.

(a) $l_{n}$ is a multiple of $\kappa$;

(b) $l_{n} \geq \tilde{b}_{n} \geq b_{n}+2 l_{n-1}$ for all $n$; and

(c) $\mu\left(\mathcal{C}_{n}^{\prime}+R_{n}^{\leftarrow \tilde{b}_{n}}\right)>1-\epsilon_{n}$ for all probability invariant measures $\mu$.

For the base of the construction, $l_{0}$ is as assumed to be zero.

Set, for all $k \geq 1$,

$$
\mathcal{C}_{k}=\mathcal{C}_{k}^{\prime} \cap\left(\bigcap_{n \geq k+1}\left(\mathcal{C}_{n}^{\prime}+R_{n}^{\leftarrow \tilde{b}_{n}-l_{k}}\right)\right),
$$

and let $Z=\bigcup_{k}\left(\mathcal{C}_{k}+R_{k}\right)$. We claim that sets $\mathcal{C}_{k}$ and $Z$ satisfy all the requirements of the theorem except, possibly, item (i). (It will be easy to enlarge sets $\mathcal{C}_{n}$ to fulfill this item.)

Items (ii)-(v) are evident from the construction. We check (vi) next. Pick $x \in \mathcal{C}_{k}$ and note that, by the definition of $\mathcal{C}_{k}$, there exists $y \in \mathcal{C}_{k+1}^{\prime}$ such that

$$
x \in y+R_{k+1}^{\leftarrow \tilde{b}_{k+1}-l_{k}} \text {. }
$$

Since $\tilde{b}_{k+1} \geq b_{k+1}+2 l_{k}$, using (5), we conclude that

$$
x+R_{k} \subseteq y+R_{k+1}^{\leftarrow \tilde{b}_{k+1}-l_{k}}+R_{k} \subseteq y+R_{k+1}^{\leftarrow \tilde{b}_{k+1}-2 l_{k}} \subseteq y+R_{k+1}^{\leftarrow b_{k+1}} .
$$

To verify (vi) it is therefore enough to check that this $y \in \mathcal{C}_{k+1}^{\prime}$ is actually an element of $\mathcal{C}_{k+1}$. Pick $n>k+1$; we show $y \in \mathcal{C}_{n}^{\prime}+R_{n}^{\leftarrow \tilde{b}_{n}-l_{k+1}}$. Since $x \in \mathcal{C}_{k}$, there exists $z \in \mathcal{C}_{n}^{\prime}$ such that

$$
x \in z+R_{n}^{\leftarrow \tilde{b}_{n}-l_{k}} .
$$

Using $\tilde{b}_{k+1}-2 l_{k} \geq 0$ and equations (6) and (7), we have the chain of inclusions

$$
\begin{aligned}
& y \in z+R_{n}^{\leftarrow \tilde{b}_{n}-l_{k}}-R_{k+1}^{\leftarrow \tilde{b}_{k+1}-l_{k}} \subseteq z+R_{n}^{\leftarrow \tilde{b}_{n}-l_{k}-\left(l_{k+1}-\tilde{b}_{k+1}+l_{k}\right)} \\
& \subseteq z+R_{n}^{\leftarrow \tilde{b}_{n}-l_{k+1}+\tilde{b}_{k+1}-2 l_{k}} \\
& \subseteq z+R_{n}^{\leftarrow \tilde{b}_{n}-l_{k+1}} \text {. }
\end{aligned}
$$


Thus $y \in \mathcal{C}_{n}^{\prime}+R_{n}^{\leftarrow \tilde{b}_{n}-l_{k+1}}$ for all $n>k+1$, and therefore $y \in \mathcal{C}_{k}$, as required. This checks (vi).

Note that (vi) implies that $Z=\bigcup_{k}\left(\mathcal{C}_{k}+R_{k}\right)$ is an invariant subset of $X$. Indeed, for any $c \in \mathcal{C}_{k}$ and any $x \in c+\mathbb{R}^{d}$, we may find $n \geq k$ so large that $x \in c+R_{n}$ (recall that we assume $\lim b_{n}=\infty$ and therefore also $\lim l_{n}=\infty$ ). By item (vi),

$$
c \in \mathcal{C}_{k+1}+R_{k+1}^{\leftarrow b_{k+1}} \subseteq \mathcal{C}_{k+2}+R_{k+2}^{\leftarrow b_{k+1}+b_{k+2}} \subseteq \cdots \subseteq \mathcal{C}_{k+m}+R_{k+m}^{\leftarrow \sum_{i=1}^{m} b_{k+i}} .
$$

For $m$ so large that $\sum_{i=1}^{m} b_{k+i} \geq l_{n}$,

$$
x \in c+R_{n} \subseteq \mathcal{C}_{k+m}+R_{k+m}^{\leftarrow \sum_{i=1}^{m} b_{k+i}-l_{n}} \subseteq \mathcal{C}_{k+m}+R_{k+m} \subseteq Z
$$

To see item (vii), note first that, for any $k$,

$$
\begin{aligned}
\bigcap_{n \geq k}\left(\mathcal{C}_{n}^{\prime}+R_{n}^{\leftarrow \tilde{b}_{n}}\right) & \subseteq\left(\mathcal{C}_{k}^{\prime}+R_{k}\right) \cap\left(\bigcap_{n \geq k+1}\left(\mathcal{C}_{n}^{\prime}+R_{n}^{\leftarrow \tilde{b}_{n}}\right)\right) \\
& \subseteq\left(\mathcal{C}_{k}^{\prime} \cap\left(\bigcap_{n \geq k+1}\left(\mathcal{C}_{n}^{\prime}+R_{n}^{\leftarrow \tilde{b}_{n}-l_{k}}\right)\right)\right)+R_{k} \\
& =\mathcal{C}_{k}+R_{k} .
\end{aligned}
$$

And, in particular, for all $k \geq 1$ and all measures $\mu$,

$$
\mu\left(\bigcap_{n \geq k}\left(\mathcal{C}_{n}^{\prime}+R_{n}^{\leftarrow \tilde{b}_{n}}\right)\right) \leq \mu\left(\mathcal{C}_{k}+R_{k}\right)
$$

Hence by item (c) in the construction of $\mathcal{C}_{k}$,

$$
\begin{aligned}
\mu(Z) & =\mu\left(\bigcup_{k}\left(\mathcal{C}_{k}+R_{k}\right)\right) \\
& \geq \sup _{k} \mu\left(\mathcal{C}_{k}+R_{k}\right) \\
& \geq \sup _{k} \mu\left(\bigcap_{n \geq k}\left(\mathcal{C}_{n}^{\prime}+R_{n}^{\leftarrow \tilde{b}_{n}}\right)\right) \\
& \geq \lim _{k \rightarrow \infty}\left(1-\sum_{n=k}^{\infty} \epsilon_{k}\right) \\
& =1 .
\end{aligned}
$$

This almost finishes the proof of the theorem. The only problem is item (i). It is possible to have points $c \in \mathcal{C}_{n}$ such that the rectangle $c+R_{n}$ has no points from $\mathcal{C}_{k}$ for $k<n$. The easy fix is to add all such $c \in \mathcal{C}_{n}$ to $\mathcal{C}_{k}$ for $k<n$ : i.e., we set

$$
\overline{\mathcal{C}}_{k}=\mathcal{C}_{k} \cup \bigcup_{n \geq k+1}\left\{z \in \mathcal{C}_{n}: \mathcal{C}_{m} \cap\left(z+R_{n}\right)=\varnothing \forall m<n\right\} .
$$

This enlargement does not violate any of the items (ii)-(vii), and sets $\overline{\mathcal{C}}_{k}$ are as desired. 


\section{Rudolph's regular tilings}

In this section we employ Theorem 6.3 and construct a regular tiling of a subspace $Z \subseteq X$ of uniformly full measure. Regularity refers to the fact that orbits will be tiled by rectangles of a finite number of shapes. To describe possible tiles, we first pick an irrational number $\alpha>0$ : for instance, $\alpha=\sqrt{2}$ will be good enough. For a vector $\vec{a} \in\{1, \alpha\}^{d}$ let

$$
\widetilde{R}_{\vec{a}}=\prod_{i=1}^{d}[-a(i) / 2, a(i) / 2) .
$$

In other words, each edge of $\widetilde{R}_{\vec{a}}$ has length one or $\alpha$. We let $\overrightarrow{\mathbf{1}}$ denote the vector $(1, \ldots, 1) \in\{1, \alpha\}^{d}$, and $\widetilde{R}_{\overrightarrow{1}}$ is therefore a square with side one.

Using Theorem 6.3, one can extract the following result from [Rud88, §3].

THEOREM 7.1. (Essentially Rudolph) Given a free Borel flow $\mathbb{R}^{d} \curvearrowright X$ and an irrational $\alpha>0$, there exists an invariant subset $Z \subseteq X$ of uniformly full measure such that, for the restriction of the action $\mathbb{R}^{d} \curvearrowright Z$, the following holds. There exists a rectangular tiling $\mathscr{R}$ of $\mathbb{R}^{d} \curvearrowright Z$ with associated cross section $\mathcal{C}$, a Borel partition $\mathcal{C}=\bigsqcup_{\vec{a} \in\{1, \alpha\}^{d}} \mathcal{C}_{\vec{a}}$ and Borel bijections $\theta_{\vec{a}}: \mathcal{C}_{\overrightarrow{\mathbf{1}}} \rightarrow \mathcal{C}_{\vec{a}}, \vec{a} \in\{1, \alpha\}^{d} \backslash\{\overrightarrow{\mathbf{1}}\}$ such that, for $\mathscr{R}_{c}=c+R_{c}$ :

(i) $\quad R_{c}=\widetilde{R}_{\vec{a}}$ for all $c \in \mathcal{C}_{\vec{a}}$; and

(ii) $\quad c E_{\mathcal{C}} \theta_{\vec{a}}(c)$ for all $c \in \mathcal{C}$ and all $\vec{a} \in\{1, \alpha\}^{d} \backslash\{\overrightarrow{\mathbf{1}}\}$.

This theorem asserts that we can find a tiling of $Z$ which uses only $2^{d}$ different tiles. It is generally easy to construct tilings with approximate properties of tiles, but getting exact restrictions on length of edges is typically a more difficult task. Naturally, we would want to have such a tiling on all $X$, but at present, it is open as to whether this can always be achieved.

During the construction of the tiling, we shall ensure that each type of tile occurs on each orbit an infinite number of times. In fact, we shall have a Borel witness for that, namely, Borel matchings $\theta_{\vec{a}}: \mathcal{C}_{\overrightarrow{\mathbf{1}}} \rightarrow \mathcal{C}_{\vec{a}}$ between tiles of type $\overrightarrow{\mathbf{1}}$ and of type $\vec{a}$ within each orbit. Figure 7 shows how such a tiling may look.

While the construction in [Rud88, §3] is presented relative to single measure $\mu$ on $X$, it only uses existence of exhausting towers given in Theorem 6.3. Existence of Borel correspondences $\theta_{\vec{a}}$ is almost immediate from the construction. For the convenience of the reader, we present a sketch of the argument. While we believe that the reader will have no difficulty in supplying the necessary details, if needed, the rigorous proof can be found in [Rud88, §3].

Sketch of Proof. The starting point is to notice that irrationality of $\alpha$ implies that the set of points

$$
\left\{m_{1}+m_{2} \alpha \mid m_{1}, m_{2} \in \mathbb{N}\right\}
$$

is asymptotically dense in the real line $\mathbb{R}$ in the sense that, for any $\epsilon>0$, there exists $N(\epsilon)$ such that for any $x \geq N(\epsilon)$ there are $m_{1}, m_{2} \in \mathbb{N}$ for which $\left|x-\left(m_{1}+m_{2} \alpha\right)\right|<\epsilon$. Geometrically, this means that any sufficiently long interval, after being perturbed by a small $\epsilon$, can be partitioned into subintervals of length one or $\alpha$. 


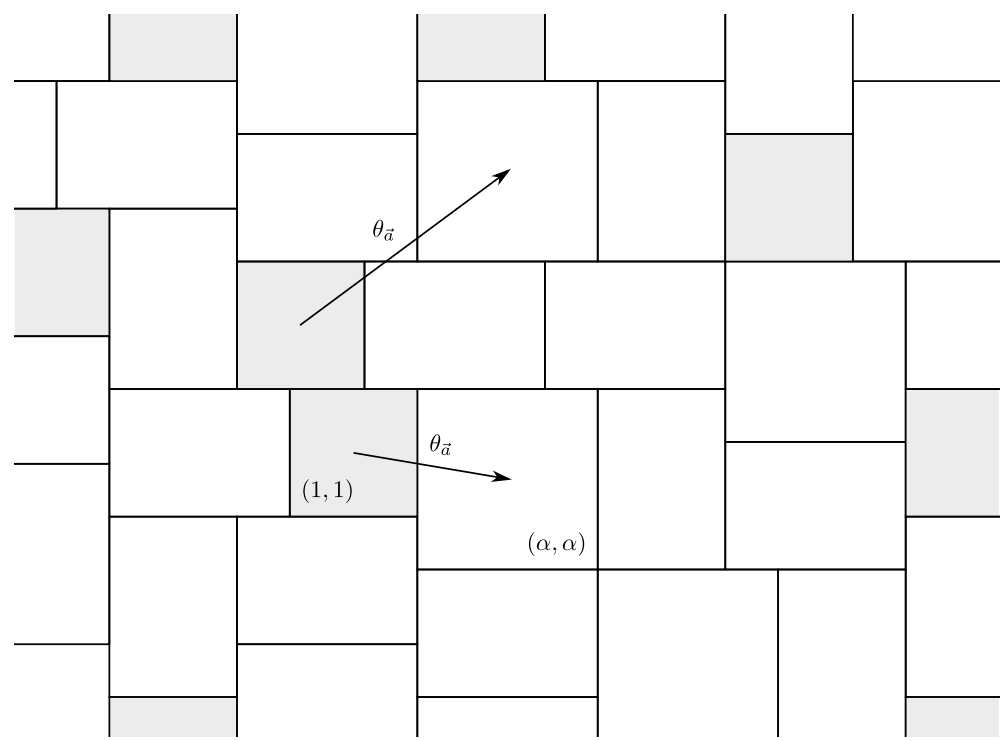

FIGURE 7. Regular tiling of an orbit. There are four types of tiles. The map $\theta_{\vec{a}}, \vec{a}=(\alpha, \alpha)$, is a match between $(1,1)$-tiles (which are in gray) and $(\alpha, \alpha)$-tiles.

Imagine now the following situation depicted in Figure 8. Suppose we have a square $R$ with side $K(1+\alpha)$ for some integer $K$. Suppose, also, that $R$ sits inside a much larger square $R^{\prime}$ with side length $K^{\prime}(1+\alpha)$ for some (much larger) integer $K^{\prime}$; suppose furthermore that the distance from $R$ to the boundary of $R^{\prime}$ is at least $N(\epsilon)$ in every coordinate direction.

For notational convenience, let us place the origin at the bottom left-hand corner of $R^{\prime}$, so $R^{\prime}=\left[0, K^{\prime}+K^{\prime} \alpha\right)^{d}$, and let

$$
R=\prod_{i=1}^{d}\left[a_{i}, b_{i}\right), \quad 0<a_{i}<b_{i}<K^{\prime}+K^{\prime} \alpha .
$$

Since we assume that $R$ is far from the boundary of $R^{\prime}, a_{i} \geq N(\epsilon)$. We therefore may move $R$ along the $x$-axis by a small $\delta_{1},\left|\delta_{1}\right| \leq \epsilon$ in such a way that $a_{1}+\delta_{1}=m_{1}+m_{2} \alpha$ for some $m_{1}, m_{2} \in \mathbb{N}$. The interval $I_{1}=\left[0, a_{1}+\delta_{1}\right)$ may thus be tiled by intervals of length one and $\alpha$. The interval

$$
I_{2}=\left[a_{1}+\delta_{1}, a_{1}+\delta_{1}+K+K \alpha\right)=\left[a_{1}+\delta, b_{1}+\delta_{1}\right)
$$

can also be tiled in such a way, since we assume that sides of $R$ have length $K(1+\alpha)$. Finally, the interval

$$
I_{3}=\left[a_{1}+\delta_{1}+K+K \alpha, K^{\prime}+K^{\prime} \alpha\right)
$$

may be partitioned into segments of length one and $\alpha$ since $R^{\prime}$ is supposed to have length $K^{\prime}+K^{\prime} \alpha$; in particular,

$$
K^{\prime}+K^{\prime} \alpha-a_{1}-\delta_{1}-K-K \alpha=\tilde{m}_{1}+\tilde{m}_{2} \alpha
$$

for some integers $\tilde{m}_{1}, \tilde{m}_{2} \in \mathbb{N}$. 

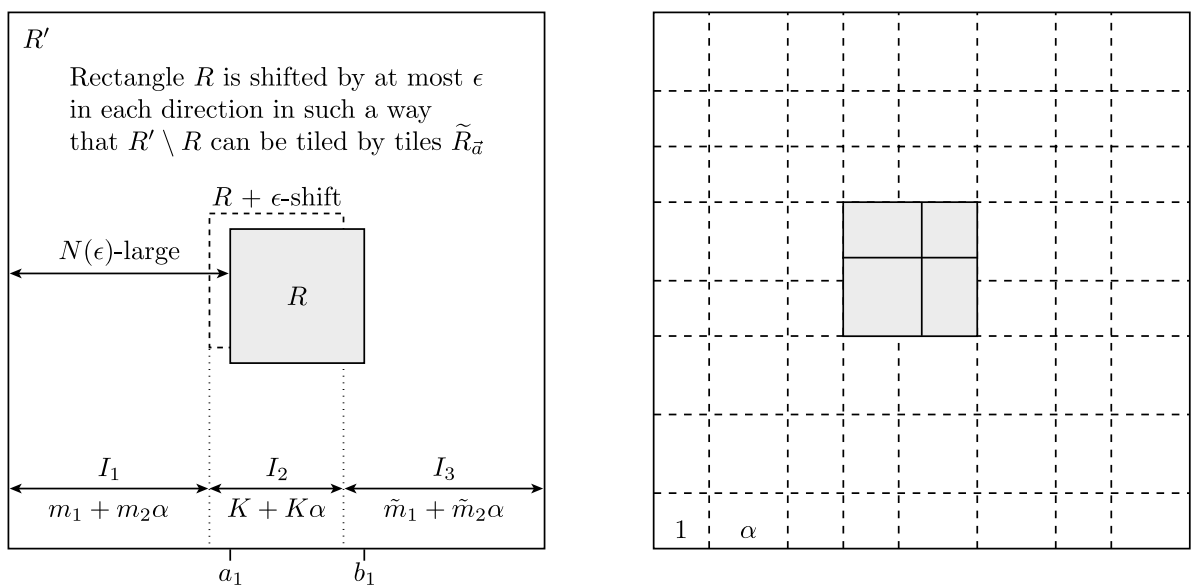

FIGURE 8. Moving the rectangle $R$ and extending the tiling to $R^{\prime}$.

The same can be done along other coordinate directions, and one may find a vector $\vec{v}$ of $\ell^{\infty}$-norm at most $\epsilon$ such that, once $R$ is shifted to $R+\vec{v}$, the rectangle $R^{\prime}$ can be tiled by regular tiles $\widetilde{R}_{\vec{a}}, \vec{a} \in\{1, \alpha\}^{d}$, in a way that is consistent with the rectangle $R+\vec{v}$.

To summarize, given a square $R$ of length $K(1+\alpha)$ which is tiled by rectangles $\widetilde{R}_{\vec{a}}$, $\vec{a} \in\{1, \alpha\}^{d}$, and which is $N(\epsilon)$-far from the boundary of $R^{\prime}$, we may shift $R$ by an $\epsilon$-small vector and extend the tiling of (shifted) $R$ to a regular tiling of $R^{\prime}$. The right-hand square in Figure 8 shows how such an extension may look.

Now to the construction of the regular tiling. Let $\left(\epsilon_{k}\right)_{k=1}^{\infty}$ be a sufficiently fast decreasing sequence, e.g., $\epsilon_{k}=2^{-k}$; let $N\left(\epsilon_{k}\right) \in \mathbb{R}^{>0}$ be so large that for any $x \geq N\left(\epsilon_{k}\right)$ there exists $m_{1}, m_{2} \in \mathbb{N}$ such that

$$
\left|x-m_{1}-m_{2} \alpha\right|<\epsilon_{k} .
$$

Pick a sequence $\left(b_{k}\right)_{k=1}^{\infty}$ such that:

(a) $b_{k}$ is a multiple of $(1+\alpha)$; and

(b) $b_{k} \geq N\left(\epsilon_{k}\right)+2(1+\alpha)$.

An application of Theorem 6.3 allows us to find the following objects:

- $\quad$ an invariant subset $Z \subseteq X$ of uniformly full measure;

- $\quad$ an increasing sequence $\left(l_{k}\right)_{k=1}^{\infty}$ of reals $l_{k} \geq b_{k}$, where each $l_{k}$ is a multiple of $(1+$ $\alpha)$; and

- $\quad$ Borel $R_{k}$-lacunary cross sections $\mathcal{C}_{k} \subseteq Z$ such that $\mathcal{C}_{k}+R_{k} \subseteq \mathcal{C}_{k+1}+R^{\leftarrow b_{k}}$, where $R_{k}=\left[-l_{k}, l_{k}\right)^{d}$.

Our plan is to inductively tile regions $\mathcal{C}_{k}+R_{k}^{\leftarrow b_{k}}$. At step $k+1$ of the construction we may shift tiles in $\mathcal{C}_{k}+R_{k}^{\leftarrow b_{k}}$ by at most $\epsilon_{k}$, thus ensuring that the sum of all shifts is finite, and each tile converges to a limiting position as $k \rightarrow \infty$.

Observe that, since $b_{k}$ and $l_{k}$ are multiples of $(1+\alpha)$, sides of $R^{\leftarrow b_{k}}$ are also multiples of $(1+\alpha)$. Therefore $R^{\leftarrow b_{k}}$ can be partitioned into tiles $\widetilde{R}_{\vec{a}}$ in a canonical fashion by partitioning each side of $R^{\leftarrow b_{k}}$ into consecutive intervals of length one and $\alpha$ and taking the product of these partitions (see Figure 9). We shall refer to this partition as the canonical tiling of $R^{\leftarrow b_{k}}$. 
In particular, for the base of our construction, each square $c+R_{1}^{\leftarrow b_{1}}$ in $\mathcal{C}_{1}+R_{1}^{\leftarrow b_{1}}$ can be tiled in this canonical way.

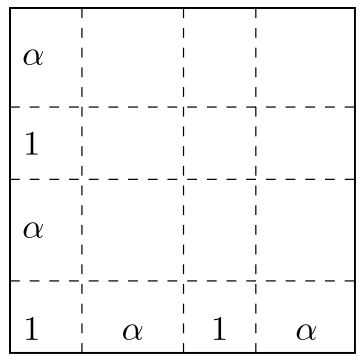

FIGURE 9. Canonical tiling.

For the induction step, we have tiled $\mathcal{C}_{k}+R_{k}^{\leftarrow b_{k}}$, and proceed to tile $\mathcal{C}_{k+1}+R_{k+1}^{\leftarrow b_{k+1}}$. Pick some $c \in \mathcal{C}_{k+1}$, and consider the corresponding square $c+R_{k+1}^{\leftarrow b_{k+1}}$. In general, it contains several points $c_{1}, \ldots, c_{m} \in \mathcal{C}_{k}$. Each square $c_{i}+R_{k}^{\leftarrow b_{k}}$ has been tiled, and we seek an extension of this tiling to a tiling of $c+R_{k}^{\leftarrow b_{k+1}}$. It is helpful to consult at this point Figure 10, on which $m=3$ and three $R_{k}^{\leftarrow b_{k}}$ rectangles are marked in gray. By assumption, squares $c_{i}+R_{k}$ are all inside $c+R_{k+1}^{\leftarrow b_{k+1}}$ and have pairwise empty intersections. The dashed lines in Figure 10 show that $R_{k+1}^{\leftarrow b_{k+1}}$ admits the canonical tiling. We shall use it to 'feel the gaps' between squares $R_{k}$. Now comes the crucial idea in the construction. One realizes that it is always possible to move squares $R_{k}$ around $c_{i}$ by at most $1+\alpha$ in such a way that corners of $R_{k}$ will coincide with nodes of the canonical tiling of $R_{k+1}^{\leftarrow b_{k+1}}$. We emphasize that tiles of $\mathcal{C}_{k}+R_{k}^{\leftarrow b_{k}}$ constructed up to this stage are not being moved, we are rather claiming that one may select 'windows' of size $R_{k}$ around each point $c_{i}$ with corners on the nodes of the canonical tiling of $R_{k+1}^{\leftarrow b_{k+1}}$ and these 'windows' are no further than $1+\alpha$ in each coordinate direction from $c_{i}+R_{k}$. It is easy to see that such squares may be chosen to be disjoint for distinct $c_{i}$. These windows $R_{k}$ are depicted on Figure 10 around each $R_{k}^{\leftarrow b_{k}}$.

Now we are going to move each $R_{k}^{\leftarrow b_{k}}$ region by a vector of $\ell^{\infty}$-norm at most $\epsilon_{k}$; according to the algorithm described at the beginning of this sketch, for each $c_{i}$ one can find $\vec{v}_{i} \in \mathbb{R}^{d},\left\|\vec{v}_{i}\right\|_{\infty}<\epsilon_{k}$, such that the tiling of $c_{i}+\vec{v}_{i}+R_{k}^{\leftarrow b_{k}}$ can be extended to the tiling of the window $R_{k}$ around $c_{i}$. On Figure 10, the bottom $R_{k}$ exhibits this process. Finally, the gaps between $R_{k}$ are filled by the canonical tiling, which extends the tiling of each $R_{k}$ to a tiling of $R_{k+1}^{\leftarrow b_{k+1}}$. To summarize, when extending the tiling of $\mathcal{C}_{k}+R_{k}^{\leftarrow b_{k}}$ to a tiling of $\mathcal{C}_{k+1}+R_{k+1}^{\leftarrow b_{k+1}}$, we shift each $c \in \mathcal{C}_{k}$ (together with all the tiles in $c+R_{k}^{\leftarrow b_{k}}$ ) by no more than $\epsilon_{k}$ in each coordinate direction. This describes the step of induction.

While, strictly speaking, the tiling of $\mathcal{C}_{k+1}+R_{k+1}^{\leftarrow b_{k+1}}$ extends the tiling of $\mathcal{C}_{k}+R_{k}^{\leftarrow b_{k}}$ only up to an $\epsilon_{k}$-shift, using $\sum_{k} \epsilon_{k}<\infty$ we may naturally define the limit tiling of $\bigcup_{k}\left(\mathcal{C}_{k}+R_{k}^{\leftarrow b_{k}}\right)=Z$. This results in a construction of a tiling of $Z$ by tiles of the form $\widetilde{R}_{\vec{a}}, \vec{a} \in\{1, \alpha\}^{d}$, as in Figure 7 . 


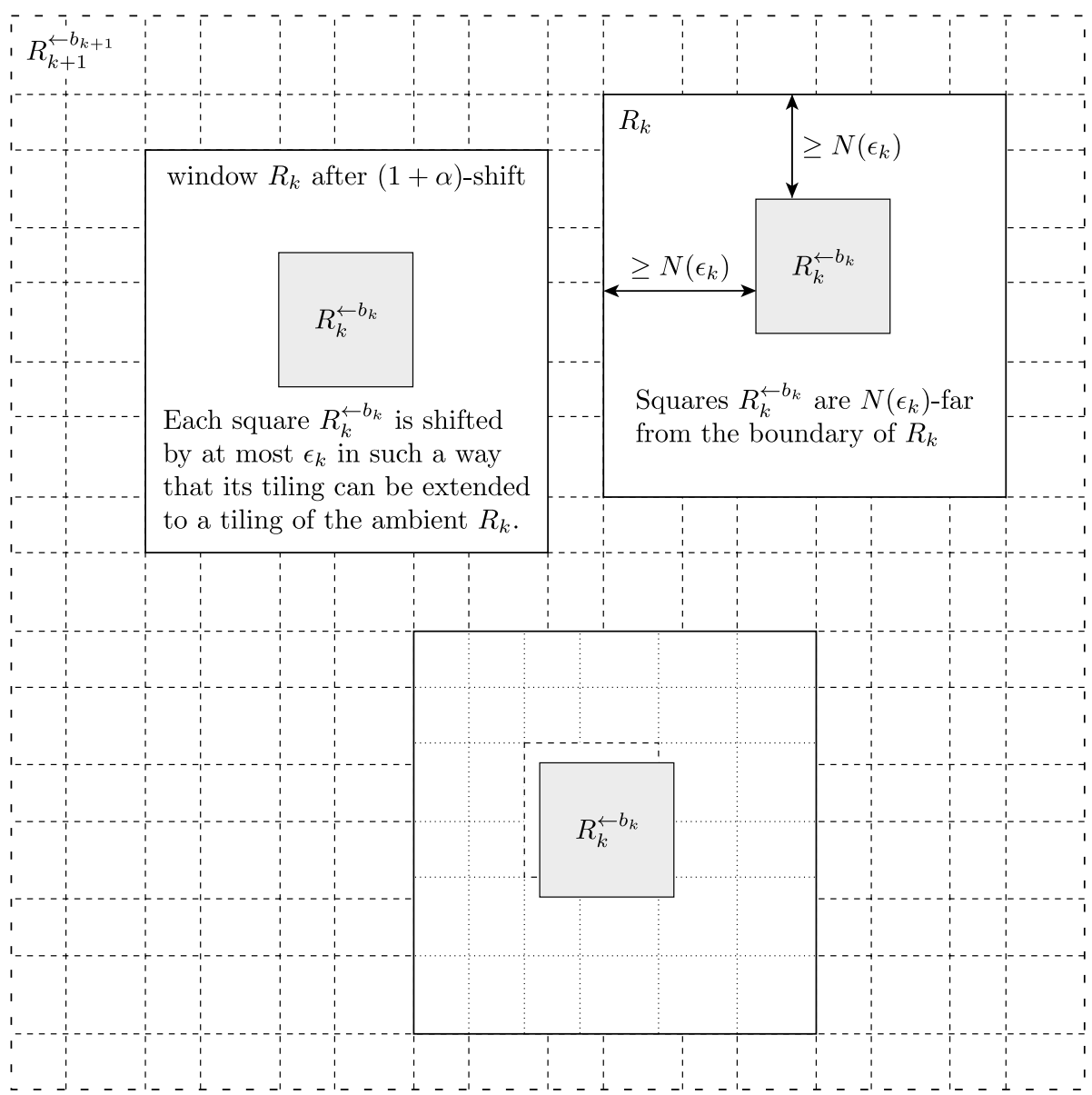

FIGURE 10. Extending tiling from $\mathcal{C}_{n}+R_{n}^{\leftarrow b_{n}}$ to $\mathcal{C}_{n+1}+R_{n+1}^{\leftarrow b_{n+1}}$.

So, let $\widetilde{\mathcal{C}}$ denote the set of centers of all the tiles in $Z$, and let

$$
\widetilde{\mathcal{C}}=\bigsqcup_{\vec{a} \in\{1, \alpha\}^{d}} \widetilde{\mathcal{C}}_{\vec{a}}
$$

be the decomposition of tiles into the $2^{d}$ types, according to lengths of their sides.

It remains to explain how the maps $\theta_{\vec{a}}: \widetilde{\mathcal{C}}_{\overrightarrow{\mathbf{1}}} \rightarrow \widetilde{\mathcal{C}}_{\vec{a}}$ are constructed. It is immediate from the construction that, for any $c \in \mathcal{C}_{k}$, the tiling of $c+R_{k}^{\leftarrow b_{k}}$ has an equal number of tiles of each type. For a given $c \in \mathcal{C}_{k+1}$, if $\theta_{\vec{a}}$ is defined on each $c_{i}+R_{k}^{\leftarrow b_{k}}, c_{i} \in \mathcal{C}_{k} \cap(c+$ $\left.R_{k+1}^{\leftarrow b_{k+1}}\right)$, then in $\left(c+R_{k+1}^{\leftarrow b_{k+1}}\right) \backslash\left(\mathcal{C}_{k}+R_{k}\right)$ we have the same number of tiles of each type, and therefore the map $\theta_{\vec{a}}$ can be extended to a matching between $\overrightarrow{\mathbf{1}}$-tiles and $\vec{a}$-tiles on $c+R_{k+1}^{\leftarrow b_{k+1}}$ in a Borel way. In the limit, $\theta_{\vec{a}}$ is a matching from $\widetilde{\mathcal{C}_{\overrightarrow{\mathbf{1}}}}$ onto $\widetilde{\mathcal{C}_{\vec{a}}}$, as desired.

We conclude this section by showing how the above theorem gives rise to a LOE between invariant subsets of uniformly full measure. 
THEOREM 7.2. Let $\mathbb{R}^{d} \curvearrowright X$ and $\mathbb{R}^{d} \curvearrowright Y$ be a pair of free Borel flows having the same cardinality of the sets of pie measures. There exist invariant Borel subsets $Z_{X} \subseteq X$ and $Z_{Y} \subseteq Y$ of uniformly full measure and a LOE $\phi: Z_{X} \rightarrow Z_{Y}$ between restrictions of the flows.

Proof. If the flows admit no pie measures, the statement is vacuously true, since one may take $Z_{X}=\varnothing=Z_{Y}$. We therefore assume that flows have invariant measures. One starts with Theorem 7.1 to find $Z_{X} \subseteq X$ and $Z_{Y} \subseteq Y$ of uniformly full measure together with regular tilings associated with cross sections $\mathcal{C}_{X} \subseteq Z_{X}$ and $\mathcal{C}_{Y} \subseteq Z_{Y}$. Notice that $|\mathcal{E}(X)|=$ $\left|\mathcal{E}\left(Z_{X}\right)\right|$ and $|\mathcal{E}(Y)|=\left|\mathcal{E}\left(Z_{Y}\right)\right|$, because $X \backslash Z_{X}$ and $Y \backslash Z_{Y}$ have measure zero with respect to all invariant measures.

We would like to apply DJK classification of hyperfinite equivalence relations to cross sections $\mathcal{C}_{X, \overrightarrow{\mathbf{1}}}$ and $\mathcal{C}_{Y, \overrightarrow{\mathbf{1}}}$ and to find a Borel isomorphism between induced equivalence relations $\phi: \mathcal{C}_{X, \overrightarrow{\mathbf{1}}} \rightarrow \mathcal{C}_{Y, \overrightarrow{\mathbf{1}}}$. For this we need to check that the equivalence relations on these cross sections possess the same number of pie measures. While Proposition 4.4 shows that $\left|\mathcal{E}\left(Z_{X}\right)\right|=\left|\mathcal{E}\left(\mathcal{C}_{X}\right)\right|$, it is not immediately clear whether this proposition can be applied to the sub cross section $\mathcal{C}_{X, \overrightarrow{\mathbf{1}}}$, as it is not evident from the construction of Theorem 7.1 whether $\mathcal{C}_{X, \overrightarrow{\mathbf{1}}}$ is cocompact in $Z_{X}$. While it is possible to modify the argument in Theorem 7.1 to ensure cocompactness of all $\mathcal{C}_{\vec{a}}$, we may show $\left|\mathcal{E}\left(\mathcal{C}_{X}\right)\right|=\left|\mathcal{E}\left(\mathcal{C}_{X, \overrightarrow{1}}\right)\right|$ instead, as follows. Since all the matchings $\theta_{\vec{a}}^{X}: \mathcal{C}_{X, \overrightarrow{\mathbf{1}}} \rightarrow \mathcal{C}_{X, \vec{a}}$ preserve the equivalence relation $E_{X}$, they also preserve all the invariant measures on $\mathcal{C}_{X}$. So, if $\mu$ is an invariant probability measure on $\mathcal{C}_{X}$, then $\mu\left(\mathcal{C}_{X}\right)=2^{d} \mu\left(\mathcal{C}_{X, \overrightarrow{\mathbf{1}}}\right)$ and $\left.2^{d} \mu\right|_{\mathcal{C}_{X, \overrightarrow{\mathbf{1}}}}$ is an invariant probability measure on $\mathcal{C}_{X, \overrightarrow{\mathbf{1}}}$. On the other hand, if $v$ is an invariant measure on $\mathcal{C}_{X, \overrightarrow{\mathbf{1}}}$, then

$$
\tilde{v}=2^{-d} \sum_{\vec{a} \in\{1, \alpha\}^{d}}\left(\theta_{\vec{a}}^{X}\right)_{*} v \quad \text { where } \theta_{\overrightarrow{\mathbf{1}}}^{X}=\mathrm{id},
$$

is easily seen to be an invariant measure on $\mathcal{C}_{X}$. These maps, $\left.\mu \mapsto 2^{d} \mu\right|_{\mathcal{C}_{X, \mathbf{1}}}$ and $v \mapsto \tilde{v}$, are inverses of each other and are bijections between $\mathcal{E}\left(\mathcal{C}_{X}\right)$ and $\mathcal{E}\left(\mathcal{C}_{X, \overrightarrow{\mathbf{1}}}\right)$. We conclude that $\left|\mathcal{E}\left(\mathcal{C}_{X}\right)\right|=\left|\mathcal{E}\left(\mathcal{C}_{X, \overrightarrow{\mathbf{1}}}\right)\right|$ and therefore

$$
\left|\mathcal{E}\left(\mathcal{C}_{X, \overrightarrow{\mathbf{1}}}\right)\right|=\left|\mathcal{E}\left(Z_{X}\right)\right|=\left|\mathcal{E}\left(Z_{Y}\right)\right|=\left|\mathcal{E}\left(\mathcal{C}_{Y, \overrightarrow{\mathbf{1}}}\right)\right| .
$$

This allows us to apply the DJK classification and get an isomorphism between the restrictions of orbit equivalence relations $\phi: \mathcal{C}_{X, \overrightarrow{\mathbf{1}}} \rightarrow \mathcal{C}_{Y, \overrightarrow{\mathbf{1}}}$ (recall that $E_{\mathcal{C}_{X}}$ and $E_{\mathcal{C}_{Y}}$ are necessarily hyperfinite by [JKL02, Theorem 1.16]). The maps $\theta_{\vec{a}}^{X}$ and $\theta_{\vec{a}}^{Y}$ make it easy to extend $\phi$ to an isomorphism $\phi: \mathcal{C}_{X} \rightarrow \mathcal{C}_{Y}$ by setting

$$
\phi \circ \theta_{\vec{a}}^{X}(c)=\theta_{\vec{a}}^{Y} \circ \phi(c) \text { for each } \vec{a} \in\{1, \alpha\}^{d} \text { and all } c \in \mathcal{C}_{X, \overrightarrow{\mathbf{1}}} .
$$

Finally, we may extend $\phi$ linearly to a $\operatorname{LOE} \phi: Z_{X} \rightarrow Z_{Y}$. More formally, for any $x \in Z_{X}$ there exist unique $\vec{a} \in\{1, \alpha\}^{d}$ and $c \in \mathcal{C}_{X, \vec{a}}$ such that $x \in c+\widetilde{R}_{\vec{a}}$. Let $\vec{v} \in \widetilde{R}_{\vec{a}}$ be such that $c+\vec{v}=x$. The point $\phi(x)$ is defined by

$$
\phi(x)=\phi(x-\vec{v})+\vec{v} .
$$

In other words, $\phi$ maps $c+\widetilde{R}_{\vec{a}}$ onto $\phi(c)+\widetilde{R}_{\vec{a}}$ in a linear fashion. It is, of course, the crucial property of our construction that $c \in \mathcal{C}_{X, \vec{a}}$ if and only if $\phi(c) \in \mathcal{C}_{Y, \vec{a}}$. 
8. Lebesgue orbit equivalences between compressible flows

In this section, we deal with flows that have no invariant probability measures. This negative condition has a positive reformulation discovered by Nadkarni [Nad90].

THEOREM. (Nadkarni) A hyperfinite $\dagger$ Borel equivalence relation has no invariant probability measures if and only if it is compressible.

There are a number of equivalent reformulations of compressibility, but we shall adopt the following one. A countable Borel equivalence relation $E$ on a standard Borel space $\mathcal{C}$ is compressible if there exist injective homomorphisms $\tau_{n}: \mathcal{C} \rightarrow \mathcal{C}, n \in \mathbb{N}$, with disjoint images: $\tau_{m}(\mathcal{C}) \cap \tau_{n}(\mathcal{C})=\varnothing$ when $m \neq n$ and

$$
c E \tau_{n}(c) \quad \text { for all } c \in \mathcal{C} \text { and all } n \in \mathbb{N} \text {. }
$$

These homomorphisms will allow us to run a back-and-forth construction of LOE map between compressible flows.

THEOREM 8.1. If free non-smooth flows $\mathbb{R}^{d} \curvearrowright X$ and $\mathbb{R}^{d} \curvearrowright Y$ admit no invariant probability measures, then these flows are Lebesgue orbit equivalent.

Proof. We begin by applying Theorem 5.3 to pick rectangular tilings $\mathscr{R}^{X}$ and $\mathscr{R}^{Y}$ of $X$ and $Y$. We agreed earlier to pick the center of each tile as its representing point, but it is convenient to deviate from this convention here and let $\mathcal{C}_{X}$ and $\mathcal{C}_{Y}$ consist of 'bottom left' corners of the domains $\mathscr{R}_{c}^{X}$ and $\mathscr{R}_{c}^{Y}$. For any $c \in \mathcal{C}_{X} \cup \mathcal{C}_{Y}$ we therefore have a rectangle $R_{c} \subseteq \mathbb{R}^{d}$ of the form $\prod_{i=1}^{d}\left[0, \zeta_{i}(c)\right)$ such that

$$
\mathscr{R}^{X}=\left\{(x, c) \in X \times \mathcal{C}_{X}: x \in c+R_{c}\right\} \quad \text { and } \quad \mathscr{R}^{Y}=\left\{(x, c) \in Y \times \mathcal{C}_{Y}: x \in c+R_{c}\right\} .
$$

According to Theorem 5.3, we may assume that $\zeta_{i}(c) \in[4,5]$ for all $c \in \mathcal{C}_{X} \cup \mathcal{C}_{Y}$ and all $i \leq d$.

These cross sections are cocompact, and by the results from $\S 4$, equivalence relations $E_{\mathcal{C}_{X}}$ and $E_{\mathcal{C}_{Y}}$ have no invariant probability measures. By [JKL02, Theorem 1.16], these relations are also hyperfinite, and therefore, by the DJK classification, there is a Borel isomorphism $\phi: \mathcal{C}_{X} \rightarrow \mathcal{C}_{Y}$ between $E_{\mathcal{C}_{X}}$ and $E_{\mathcal{C}_{Y}}$.

In what follows, we extend $\phi$ to a $\operatorname{LOE} \phi: X \rightarrow Y$ between $E_{X}$ and $E_{Y}$.

For $k \geq 0$, let $B^{k}=\prod_{i \leq d}\left[0,2^{-k}\right)$ denote a semi-open $d$-dimensional square with side $2^{-k}$, and let us fix, for a moment, a single tile $R_{c}$ for some $c \in \mathcal{C}_{X}$. We describe a process of covering a portion of $R_{c}$ by copies of $B^{k}$. Let $n_{i, k}(c)=n_{i, k}$ be the smallest integer such that

$$
\frac{2^{-k} \cdot n_{i, k}}{\zeta_{i}(c)} \in\left(1-2^{-k-1}, 1\right) .
$$

Here is a verbose explanation of this parameter. Consider the interval $\left[0, \zeta_{i}(c)\right)$, and let us start tiling it with intervals of length $2^{-k}$ beginning from the left endpoint. The integer $n_{i, k}$ is the smallest integer such that, if we put $n_{i, k}$ many intervals $\left[0,2^{-k}\right)$ into $\left[0, \zeta_{i}(c)\right)$, then the proportion of $\left[0, \zeta_{i}(c)\right)$ that is not covered is less than $2^{-k-1}$. Since, in

$\dagger$ As proved in [BK96, Theorem 4.3.1], the assumption of hyperfiniteness may be dropped; the theorem is true for all countable Borel equivalence relations. 
our situation, $\zeta_{i}(c)$ is always between four and five, $n_{i, 0}(c)=3$ for all $i$ and all $c$, but for $k \geq 1$ the parameter will start to vary.

Note that, since $n_{i, k}$ is defined to be the smallest integer satisfying (8), in fact

$$
\frac{2^{-k} \cdot n_{i, k}}{\zeta_{i}(c)} \in\left(1-2^{-k-1}, 1-2^{-k-2}\right] \quad \text { for all } i \leq d \text { and } k \geq 0 \text {. }
$$

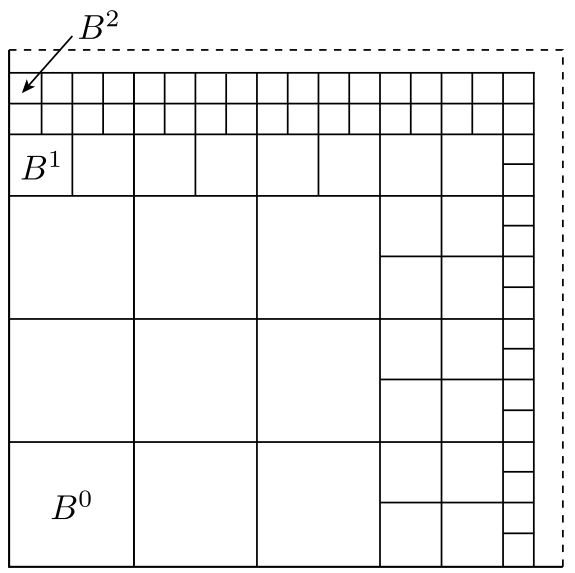

FIGURE 11. Cover of $R_{c}$ by copies of $B^{k}$.

We now partially cover $R_{c}$ with copies of $B^{k}$ by putting $n_{i, k}$ many rectangles $B^{k}$ in the $i$ th direction of $R_{c}$ starting from the 'bottom left' corner.

Figure 11 illustrates the levels $k=0,1$ and 2 of this process. On that figure, $n_{1,0}=$ $n_{2,0}=3$, and we therefore have nine squares $B^{0}$-three in each row and each column. At the level $k=1, n_{1,1}=8$ and $n_{1,1}=7$ resulting in 56 copies of $B^{1}$. Note that, in Figure 11, blocks $B^{1}$ which refine those of $B^{0}$ are not shown; only the blocks which cover parts of $R_{c}$ uncovered by $B^{0}$ are depicted. Finally, for $k=2$, we have $n_{1,2}=17$ and $n_{2,2}=16$ and blocks $B^{2}$ cover even more of $R_{c}$.

This partial cover can be constructed in a Borel fashion for all points $c$ in $\mathcal{C}_{X} \cup \mathcal{C}_{Y}$, which results in chains of Borel cross sections

$$
\mathcal{C}_{X} \subseteq \mathcal{C}_{X}^{0} \subseteq \mathcal{C}_{X}^{1} \subseteq \cdots \quad \text { and } \quad \mathcal{C}_{Y} \subseteq \mathcal{C}_{Y}^{0} \subseteq \mathcal{C}_{Y}^{1} \subseteq \cdots,
$$

where $\mathcal{C}_{X}^{k}$ consists of 'bottom left' endpoints of blocks $B^{k}$, that satisfy the following properties.

(a) $\mathcal{C}_{X}^{k}+B^{k} \subseteq \mathcal{C}_{X}^{k+1}+B^{k+1}$ - the next level of blocks covers at least as much as the previous.

(b) $\quad\left(c+R_{c}\right) \cap\left(\mathcal{C}_{X}^{k+1} \backslash\left(\mathcal{C}_{X}^{k}+B^{k}\right)\right) \neq \varnothing$ for all $c \in \mathcal{C}_{X}$-within every tile $R_{c}$ there is always a point from $\mathcal{C}_{X}^{k+1}$ which has not been covered by any $B^{k}$ block. In other words, within every tile, blocks $B^{k+1}$ cover strictly more than $B^{k}$ blocks.

(c) $\quad X=\bigcup_{k}\left(\mathcal{C}_{X}^{k}+B^{k}\right)$ - every point in $X$ is covered from some level on.

Of course, similar properties hold for $\mathcal{C}_{Y}$ instead of $\mathcal{C}_{X}$.

We are now ready to run a back-and-forth extension of $\phi: \mathcal{C}_{X} \rightarrow \mathcal{C}_{Y}$, beginning with the step $k=0$. Since we have chosen our tiles in such a way that $n_{i, 0}=3$ for all $i$, each tile $R_{c}$ has $3^{d}$ blocks $B^{0}$. 

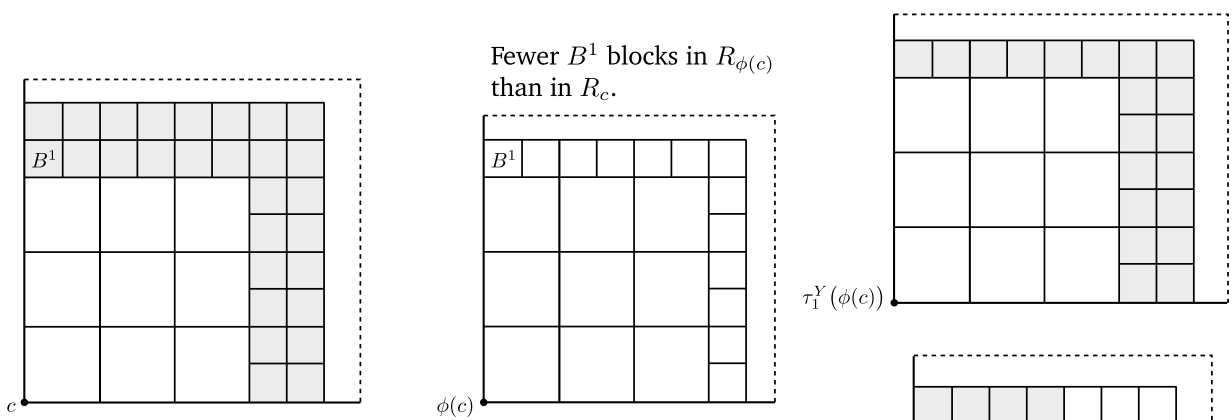

While there are not enough $B^{1}$ blocks available in $R_{\phi(c)}$, there are more $B^{1}$ blocks not in the range of $\phi$ in $R_{\tau_{1}^{Y}(\phi(c))}$ and $R_{\tau_{2}^{Y}(\phi(c))}$ combined than in $R_{c}$. Gray $B^{1}$ blocks in $R_{c}$ are mapped linearly onto gray $B^{1}$ blocks in $R_{\tau_{1}^{Y}(\phi(c))}$ and $R_{\tau_{2}^{Y}(\phi(c))}$. Note that some of the $B^{1}$ blocks in $R_{\tau_{2}^{Y}(\phi(c))}$ remain available. We shall take care of them during the 'back' stage of the construction.

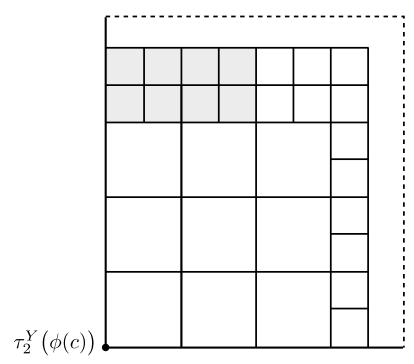

FIGURE 12. Forth step extension.

We therefore may extend $\phi$ first to a Borel isomorphism $\phi: \mathcal{C}_{X}^{0} \rightarrow \mathcal{C}_{Y}^{0}$ between $E_{\mathcal{C}_{X}^{0}}$ and $E_{\mathcal{C}_{Y}^{0}}$ by matching $\left(c+R_{c}\right) \cap \mathcal{C}_{X}^{0}$ with points in $\left(\phi(c)+R_{\phi(c)}\right) \cap \mathcal{C}_{Y}^{0}$ for all $c \in \mathcal{C}_{X}$, and then extend $\phi: \mathcal{C}_{X}^{0}+B^{0} \rightarrow \mathcal{C}_{Y}^{0}+B^{0}$ linearly on each block $B^{0}$. The map $\phi$ defined this way preserves Lebesgue measure within orbits on its domain. Since $n_{i, 0}(c)=3$ for all $c \in \mathcal{C}_{X}$ and all $i \leq d$, there is no need for the 'back' part of the argument and we proceed to the next step of the construction.

At the level $k=1$ we would like to extend $\phi$ which is currently defined on $\mathcal{C}_{X}^{0}+B^{0}$ to a map $\phi: \mathcal{C}_{X}^{1}+B^{1} \rightarrow \mathcal{C}_{Y}^{1}+B^{1}$. A naive approach would be to take $c \in \mathcal{C}_{X}$ and to try to map injectively the elements $\mathcal{C}_{X}^{1} \backslash\left(\mathcal{C}_{X}^{0}+B^{0}\right)$ from $c+R_{c}$ to corresponding elements from $\phi(c)+R_{\phi(c)}$. This approach may fail, since there may be more elements in the domain, than in the range. For instance, in the example in Figure 12 we have $n_{1,1}(c)=8$ and $n_{2,1}(c)=8$, while in the image $R_{\phi(c)}$ we may have $n_{1,1}(\phi(c))=7$ and $n_{2,1}(\phi(c))=7$, and so there will be 28 blocks $B^{1}$ in $c+R_{c}$ not in the domain of $\phi$ at the current stage, and only 13 blocks $B^{1}$ in $\phi(c)+R_{\phi(c)}$ not in the range of $\phi$.

We overcome this obstacle by using the maps $\tau_{n}^{Y}: \mathcal{C}_{Y} \rightarrow \mathcal{C}_{Y}$ which witness compressibility. By item (b), for any $\tilde{c} \in \mathcal{C}_{Y}$ there exists at least one $B^{1}$ block in $\tilde{c}+R_{\tilde{c}}$ which is not in the range of $\phi$. On the other hand, there is a uniform upper bound on the number of $B^{1}$ blocks in any $c+R_{c}$ tile, $c \in \mathcal{C}_{X}$. Therefore, for $N_{1}$ sufficiently large, the total number of $B^{1}$ blocks in tiles

$$
\tau_{1}^{Y}(\phi(c))+R_{\tau_{1}^{Y}(\phi(c))}, \ldots, \tau_{N_{1}}^{Y}(\phi(c))+R_{\tau_{N_{1}}^{Y}(\phi(c))}
$$

which are not in the range of $\phi$ exceeds the number of $B^{1}$ blocks in $c+R_{c}$. We can thus extend $\phi$ to an injective function $\phi: \mathcal{C}_{X}^{1} \rightarrow \mathcal{C}_{Y}^{1}$ by mapping, for each $c \in \mathcal{C}_{X}$, 


$$
\begin{aligned}
& \text { points in }\left(c+R_{c}\right) \cap\left(\mathcal{C}_{X}^{1} \backslash\left(\mathcal{C}_{X}^{0}+B^{0}\right)\right) \\
& \text { to points in } \bigcup_{j=1}^{N_{1}}\left(\left(\tau_{j}^{Y}(\phi(c))+R_{\tau_{j}^{Y}(\phi(c))}\right) \cap\left(\mathcal{C}_{Y}^{1} \backslash\left(\mathcal{C}_{Y}^{0}+B^{0}\right)\right)\right),
\end{aligned}
$$

and then extend it linearly to $\phi: \mathcal{C}_{X}^{1}+B^{1} \rightarrow \mathcal{C}_{Y}^{1}+B^{1}$. This finishes the 'forth' part of our back-and-forth argument.

The 'back' part of the argument is similar. At the moment, $\phi$ is defined on all $B^{1}$ blocks in $X$, while the range of $\phi$ consists of all $B^{0}$ blocks and some $B^{1}$ blocks in $Y$. In other words, $\phi^{-1}$ is defined on some of the $B^{1}$ blocks of $Y$, and we would like to extend $\phi^{-1}$ to all blocks $B^{1}$ in $Y$. We shall map $B^{1}$ blocks of $Y$ which are not yet in the domain of $\phi^{-1}$ onto $B^{2}$ blocks in $X$. Each $B^{1}$ block will be mapped (in a measure preserving way) onto $2^{d}$ blocks $B^{2}$. Since there is a uniform bound on the number of $B^{1}$ blocks in a tile $\tilde{c}+R_{\tilde{c}}$, $\tilde{c} \in \mathcal{C}_{Y}$, and since each tile $c+R_{c}, c \in \mathcal{C}_{X}$, contains at least one $B^{2}$ block not yet in the domain of $\phi$ (because of item (b) and because the domain of $\phi$ currently consists of $B^{1}$ rectangles only), there exists a sufficiently large $M_{1}$ such that, for any $\tilde{c} \in \mathcal{C}_{Y}$, the number of available $B^{2}$ blocks in the tiles

$$
\tau_{1}^{X}\left(\phi^{-1}(\tilde{c})\right)+R_{\tau_{1}^{X}\left(\phi^{-1}(\tilde{c})\right)}, \ldots, \tau_{M_{2}}^{X}\left(\phi^{-1}(\tilde{c})\right)+R_{\tau_{M_{1}}^{X}\left(\phi^{-1}(\tilde{c})\right)}
$$

exceeds

$$
2^{d} \cdot\left|\left(\tilde{c}+R_{\tilde{c}}\right) \cap \mathcal{C}_{Y}^{1}\right| .
$$

We may therefore extend $\phi$ in such a way that $\phi^{-1}$ is defined on all $\mathcal{C}_{Y}^{1}+B^{1}$, and $\phi$ preserves the Lebesgue measure on its domain. This ends the 'back' part.

The construction continues in the same fashion. The map $\phi$ is now defined on some $B^{2}$ blocks in $X$ and we extend it to all of $\mathcal{C}_{X}^{2}+B^{2}$ in such a way that the image of a $B^{2}$ block in $X$ is a $B^{2}$ block in $Y$. In general, $\phi$ will satisfy

$$
\phi\left(\mathcal{C}_{X}^{k}+B^{k}\right) \subseteq \mathcal{C}_{Y}^{k}+B^{k} \quad \text { and } \quad \phi^{-1}\left(\mathcal{C}_{Y}^{k}+B^{k}\right) \subseteq \mathcal{C}_{X}^{k+1}+B^{k+1} .
$$

From item (c), it is immediate that, in the limit, $\phi$ is a Borel isomorphism between $X$ and $Y$, and the construction ensures that $\phi: X \rightarrow Y$ is a Lebesgue orbit equivalence.

\section{Proof of the main theorem}

THEOREM 9.1. Let $\mathbb{R}^{d} \curvearrowright X$ and $\mathbb{R}^{d} \curvearrowright Y$ be a pair of free non-smooth flows. These flows are $L O E$ if and only if $|\mathcal{E}(X)|=|\mathcal{E}(Y)|$.

Proof. Necessity was proved in Theorem 4.5. We prove sufficiency. The combination of Theorems 7.2 and 8.1 almost works: we may select invariant $Z_{X} \subseteq X$ and $Z_{Y} \subseteq Y$ with a LOE $\phi: Z_{X} \rightarrow Z_{Y}$ and reduce the problem to finding a LOE between flows $\mathbb{R}^{d} \curvearrowright$ $X \backslash Z_{X}$ and $\mathbb{R}^{d} \curvearrowright Y \backslash Z_{Y}$ which have no invariant measures. The only difficulty in applying Theorem 8.1 to the latter is that $X \backslash Z_{X}$ or $Y \backslash Z_{Y}$ may be smooth. Fortunately, this obstacle is easy to overcome.

Let $X_{0} \subseteq X$ and $Y_{0} \subseteq Y$ be invariant subsets such that the restrictions $\mathbb{R}^{d} \curvearrowright X_{0}$ and $\mathbb{R}^{d} \curvearrowright Y_{0}$ are not smooth but admit no pie measures. Such subsets can be selected as follows. Pick a cocompact cross section $\mathcal{C} \subseteq X$ and select an invariant Borel subset 
$\mathcal{C}_{0} \subseteq \mathcal{C}$ such that the equivalence relation $E_{\mathcal{C}_{0}}$ is compressible, but not smooth (see, for instance, [DJK94, Corollary 7.2]). Set $X_{0}$ to be the saturation of $\mathcal{C}_{0}, X_{0}=\mathcal{C}_{0}+\mathbb{R}^{d}$. The set $Y_{0} \subseteq Y$ can be selected in a similar way.

Having picked such $X_{0}$ and $Y_{0}$, let $X^{\prime}=X \backslash X_{0}$ and $Y^{\prime}=Y \backslash Y_{0}$. Now apply Theorem 7.2 to flows $\mathbb{R}^{d} \curvearrowright X^{\prime}$ and $\mathbb{R}^{d} \curvearrowright Y^{\prime}$. As an output, we get a LOE $\phi: Z_{X^{\prime}} \rightarrow Z_{Y^{\prime}}$ between subsets of uniformly full measure. Now consider the flows restricted to the complements: that is,

$$
\mathbb{R}^{d} \curvearrowright X_{0} \cup\left(X^{\prime} \backslash Z_{X^{\prime}}\right) \text { and } \mathbb{R}^{d} \curvearrowright Y_{0} \cup\left(Y^{\prime} \backslash Z_{Y^{\prime}}\right) .
$$

These have no pie measures and are necessarily non-smooth, and hence we may apply Theorem 8.1 to extend $\phi$ to a LOE between $\mathbb{R}^{d} \curvearrowright X$ and $\mathbb{R}^{d} \curvearrowright Y$.

Acknowledgement. The author wants to thank the referee for the careful reading of the paper and numerous helpful remarks.

\section{REFERENCES}

[BK96] H. Becker and A. S. Kechris. The Descriptive Set Theory of Polish Group Actions (London Mathematical Society Lecture Note Series, 232). Cambridge University Press, Cambridge, 1996.

[DJK94] R. Dougherty, S. Jackson and A. S. Kechris. The structure of hyperfinite borel equivalence relations. Trans. Amer. Math. Soc. 341(1) (1994), 193-225.

[FM77] J. Feldman and C. C. Moore. Ergodic equivalence relations, cohomology, and von Neumann algebras. I. Trans. Amer. Math. Soc. 234(2) (1977), 289-324.

[GJ15] S. Gao and S. Jackson. Countable abelian group actions and hyperfinite equivalence relations. Invent. Math. 201(1) (2015), 309-383.

[JKL02] S. Jackson, A. S. Kechris and A. Louveau. Countable Borel equivalence relations. J. Math. Log. 2(1) (2002), 1-80.

[Kec92] A. S. Kechris. Countable sections for locally compact group actions. Ergod. Th. \& Dynam. Sys. 12(2) (1992), 283-295.

[Kec95] A. S. Kechris. Classical Descriptive Set Theory (Graduate Texts in Mathematics, 156). Springer, New York, 1995.

[KPV13] D. Kyed, H. D. Petersen and S. Vaes. $L^{2}$-betti numbers of locally compact groups and their cross section equivalence relations. Trans. Amer. Math. Soc. 367(7) (2015), 4917-4956.

[Kre76] U. Krengel. On Rudolph's representation of aperiodic flows. Ann. Inst. H. Poincaré Sect. B (N.S.) 12(4) (1976), 319-338.

[KST99] A. S. Kechris, S. Solecki and S. Todorčević. Borel chromatic numbers. Adv. Math. 141(1) (1999), $1-44$.

[Lin75] D. A. Lind. Locally compact measure preserving flows. Adv. Math. 15 (1975), 175-193.

[MR10] B. D. Miller and C. Rosendal. Descriptive Kakutani equivalence. J. Eur. Math. Soc. (JEMS) 12(1) (2010), 179-219.

[Nad90] M. G. Nadkarni. On the existence of a finite invariant measure. Proc. Indian Acad. Sci. Math. Sci. 100(3) (1990), 203-220.

[Rud79] D. J. Rudolph. Smooth orbit equivalence of ergodic $\mathbb{R}^{d}$ actions, $d \geq 2$. Trans. Amer. Math. Soc. 253 (1979), 291-302.

[Rud88] D. J. Rudolph. Rectangular tilings of $\mathbb{R}^{n}$ and free $\mathbb{R}^{n}$ actions. Dynamical Systems (College Park, MD) 1986-1987 (Lecture Notes in Mathematics, 1342). Springer, Berlin, 1988, pp. 653-688.

[Str74] R. A. Struble. Metrics in locally compact groups. Compositio Math. 28 (1974), 217-222. 